\title{
Effective strategies for childhood obesity prevention via school based, family involved interventions: a critical review for the development of the Feel4Diabetes- study school based component
}

Christina-Paulina Lambrinou', Odysseas Androutsos², Eva Karaglani', Greet Cardon³, Nele Huys ${ }^{3}$, Katja Wikström4, Jemina Kivelä ${ }^{4}$, Winne Ko ${ }^{5}$, Ernest Karuranga ${ }^{5}$, Kaloyan Tsochev ${ }^{6}$, Violeta lotova ${ }^{6}$, Roumyana Dimova ${ }^{7}$, Pilar De Miguel-Etayo ${ }^{8,9}$, Esther M. González-Gil ${ }^{8,9,10}$, Hajnalka Tamás ${ }^{11}$, Zoltán JANCSÓ ${ }^{11}$, Stavros Liatis ${ }^{12}$, Konstantinos Makrilakis ${ }^{12}$, Yannis Manios ${ }^{1 *}$ and on behalf of the Feel4Diabetes-study group

\footnotetext{
Abstract

Background: Although there are many interventions targeting childhood obesity prevention, only few have demonstrated positive results. The current review aimed to gather and evaluate available school-based intervention studies with family involvement targeting dietary, physical activity and sedentary behaviors among primary schoolchildren and their families, in order to identify the most effective strategies.

Methods: Studies published between 2000 and January 2015 were retrieved from scientific electronic databases and grey literature. The databases used included MEDLINE/PubMed, Web-of-Science, CINAHL and Scopus. Included studies had to be experimental controlled studies and had duration over 1 school year, had family involvement, combined PA and dietary behaviors and were implemented in school setting. A complementary search was executed to update the review to cover the period from February 2015 to January 2019.

(Continued on next page)
}

\footnotetext{
*Correspondence: manios@hua.gr

1 Department of Nutrition and Dietetics, School of Health Science and Education, Harokopio University, 70 El Venizelou Ave, 17671 Kallithea, Athens, Greece

Full list of author information is available at the end of the article
}

C C The Author(s). 2020 Open Access This article is licensed under a Creative Commons Attribution 4.0 International License, which permits use, sharing, adaptation, distribution and reproduction in any medium or format, as long as you give appropriate credit to the original author(s) and the source, provide a link to the Creative Commons licence, and indicate if changes were made. The images or other third party material in this article are included in the article's Creative Commons licence, unless indicated otherwise in a credit line to the material. If material is not included in the article's Creative Commons licence and your intended use is not permitted by statutory regulation or exceeds the permitted use, you will need to obtain permission directly from the copyright holder. To view a copy of this licence, visit http://creativecommons.org/licenses/by/4.0/ The Creative Commons Public Domain Dedication waiver (http://creativecommons.org/publicdomain/zero/1.0/) applies to the data made available in this article, unless otherwise stated in a credit line to the data. 


\begin{abstract}
(Continued from previous page)
Results: From the studies examined ( $n=425), 27$ intervention programs (33 publications) fulfilled the inclusion criteria. Among these, 15 presented significant effect on weight status and/ or overweight/ obesity or clinical indices, 3 presented significant effect on most energy balance-related behaviors (EBRBs) while 9 presented significant effect on some/few EBRBs or determinants. Strategies implemented in effective interventions were: teachers acting as role-models and being actively involved in the delivery of the intervention, school policies supporting the availability of healthy food and beverage choices and limiting unhealthy snacks, changes in the schoolyard, in the recess rules and in the physical education classes to increase physical activity, and involving parents in the intervention via assignments, meetings, informative material and encouraging them to improve the home environment. Use of incentives for children, social marketing techniques, collaboration with local stakeholders were found to increase effectiveness. Programs that focused only on educational sessions and material for parents, without promoting relevant environmental and policy changes, were found to be less effective. Cultural adaptations have been suggested to increase the intervention's acceptance in specific or vulnerable population groups.
\end{abstract}

Conclusions: Several effective strategies were identified in the reviewed programs. Outcomes of the current review were taken into account in developing the Feel4Diabetes-intervention and summed up as recommendations in the current work in order to facilitate other researchers designing similar childhood obesity prevention initiatives.

Keywords: Obesity prevention, Type 2 diabetes prevention, Primary school children, Families, School based intervention

\section{Background}

Childhood obesity is a growing world-wide health problem. It is estimated that about 170 million children are currently with overweight globally [1]. In US children, obesity prevalence has increased from 6\% in 1980 to $17 \%$ in 2008 [2] and to $18.5 \%$ in 2016 [3]. In Europe, the trend of childhood obesity is following the same pattern, with its prevalence increasing across most European countries [4], especially in low-to-middle-income countries (LMICs) and in vulnerable groups $[5,6]$.

Being overweight or obese has serious health consequences, especially for children. Having a high body mass index (BMI) is a major risk factor for diseases such as cardiovascular disease, type 2 diabetes and cancer later in life $[7,8]$. These diseases, also known as noncommunicable diseases (NCDs), can cause premature mortality as well as long-term morbidity. Due to the significant increase in the prevalence of obesity and the serious public health consequences, obesity is considered one of the most important public health challenges of the twenty-first century $[9,10]$.

Early childhood is considered to provide a unique opportunity to establish lifestyle behaviors such as healthy eating habits, physical activity and limited sedentary time that will promote health and minimize the risk of obesity. This is further supported by the fact that, as a results of these behaviours, childhood obesity tracks into adulthood with overweight preschool children being more likely to become overweight adults in comparison to their normal-weight peers [11-15]. Thus, interventions promoting such healthy behaviors in childhood provide a key strategy in the prevention of obesity, since it has been shown that treating obesity in adulthood poses more difficulties than changing lifestyle habits earlier on [16].

To date, many programs have been developed in order to prevent obesity in children. The vast majority of these programs use schools for the implementation of interventions [17]. Schools are considered an important setting for intervening in children's obesity related behavior, for a number of reasons: (i) primary school education is compulsory for all children in most countries and reaches all children with different backgrounds (ii) children spend a significant part of their daily life at school, usually consuming one or two meals per day; (iii) schools offer physical education classes and provide opportunities for physical activity during recess; (iv) schools offer a structured environment where interventions can be easily applied/ fit, (v) implementers can reach many children in a relatively short time via schools; (vi) teaching staff can significantly facilitate and contribute to the delivery of the intervention, thus increasing the intervention's sustainability [18]. Despite the aforementioned advantages, the overall impact of school-based interventions is questionable and generalizable recommendations cannot be easily extracted [2].

For the needs of the European multicenter Feel4Diabetes-study the current study examined interventions that have been implemented in the school setting and focused on the promotion of healthy eating and physical activity and the reduction/interruption of sedentary behavior aiming to prevent childhood obesity. The Feel4Diabetes-study was implemented in the overall population in low/middle-income countries (Bulgaria, 
Hungary), in low socio-economic areas in high-income countries (Belgium, Finland) and in countries under austerity measures (Greece, Spain). More information on this project can be found in the literature [19]. The aim of the current review was to identify the most effective strategies in improving health behaviors and tackling obesity in primary school-aged children with special emphasis given to low socioeconomic status and vulnerable groups that Feel4Diabete-study specifically targeted, taking into account their increased risk for obesity $[9,20]$ and type 2 diabetes [21]. The Feel4Diabetes project was developed using the PRECEDE-PROCEED model as the theoretical basis. The current review served as a part of the PRECEDE phase of this specific theoretical model and its outcomes were utilized in the development of the Feel4Diabetes school- and community-based intervention.

\section{Methods}

\section{Search strategy}

The bibliographic search strategy focused on articles published in peer-reviewed, English language journals, published from 2000 to January 2015, when the Feel4Diabetes-intervention was developed. A complementary search was executed to update the results for the publication to cover the period from February 2015 to January 2019 following the same methodology. The databases used included MEDLINE/PubMed, Web-ofScience, CINAHL and Scopus. Citations in reviews were also used. The PICO key terms used for conducting the literature search are presented in Supplementary Table 1.

\section{Selection criteria}

To be included, studies had to be original experimental controlled studies with school-based interventions aiming to prevent obesity in primary school-aged children from any country, published between 2000 and January 2019, in English. Additional inclusion criteria were applied based on recent literature [18, 22]. These criteria were, namely: a) duration of $>1$ school year (i.e. at least 6 months), b) family involvement, either by contacting parents via their children with the use of newsletters, etc. or by having meetings with them, c) combination of physical activity and dietary behaviors, d) implementation of the school-based intervention by school personnel (i.e. healthcare professionals working at schools or teachers). These criteria were applied in order to avoid repetition of already proven to be effective strategies and take the existing literature a step further.

\section{Exclusion criteria}

Exclusion criteria included interventions implemented in preschools, early childcare programs, adolescents or after-school programs, descriptive or analytic studies, reviews on surgical or pharmaceutical treatments, literature reviews, opinions or editorials, reports published as meeting abstracts only, reviews of causal relationships between obesity and related factors, articles reporting study design and/or process evaluation only as well as papers focused on physiological, molecular or genetic research and papers focused on morbidities (such as kidney disease and diabetes) in which obesity is a comorbidity.

\section{Selection strategy and data extraction procedures}

The flowchart of the publication identification process is presented in Fig. 1. Data from the included studies were extracted using a standardized form. Extracted data included: author, year of publication, project name, country, participant data, intervention duration, theory used and strategies implemented as described in the methodology of the relevant publications, outcomes as well as follow-up period if available. In addition, the setting in which the intervention was implemented was also extracted in order to identify strategies that proved to be effective in low-SES areas or in vulnerable groups. A summary table of the selected studies was constructed and the clinical significance of the results was evaluated for each study, to facilitate interpretation of the effectiveness of intervention. The scoring is marked in the summary table as follows:

- $(+++)$ significant effect on weight status and/ or overweight/ obesity or clinical indices.

- (++) significant improvement in most important target behaviors.

- (+) significant improvement in some/few secondary target behaviors.

- (-) no effect.

\section{Results}

The studies included in the current analysis have been summarized in Table 1 outlining the target population, intervention strategies and design, measures, outcomes, and findings relevant to obesity prevention. The interventions in table have been arranged alphabetically based on first author's name but with clustering the publications of same projects together.

\section{General description of findings}

Using the aforementioned search strategy 1148 publications were identified. After removing duplicates and a first title screening 425 articles remained. These 425 articles were screened based on their abstract, in order to discard studies without yet published results, those targeting specific populations (e.g. only obese children or patients), preschoolers or adolescents. Of the 122 articles 


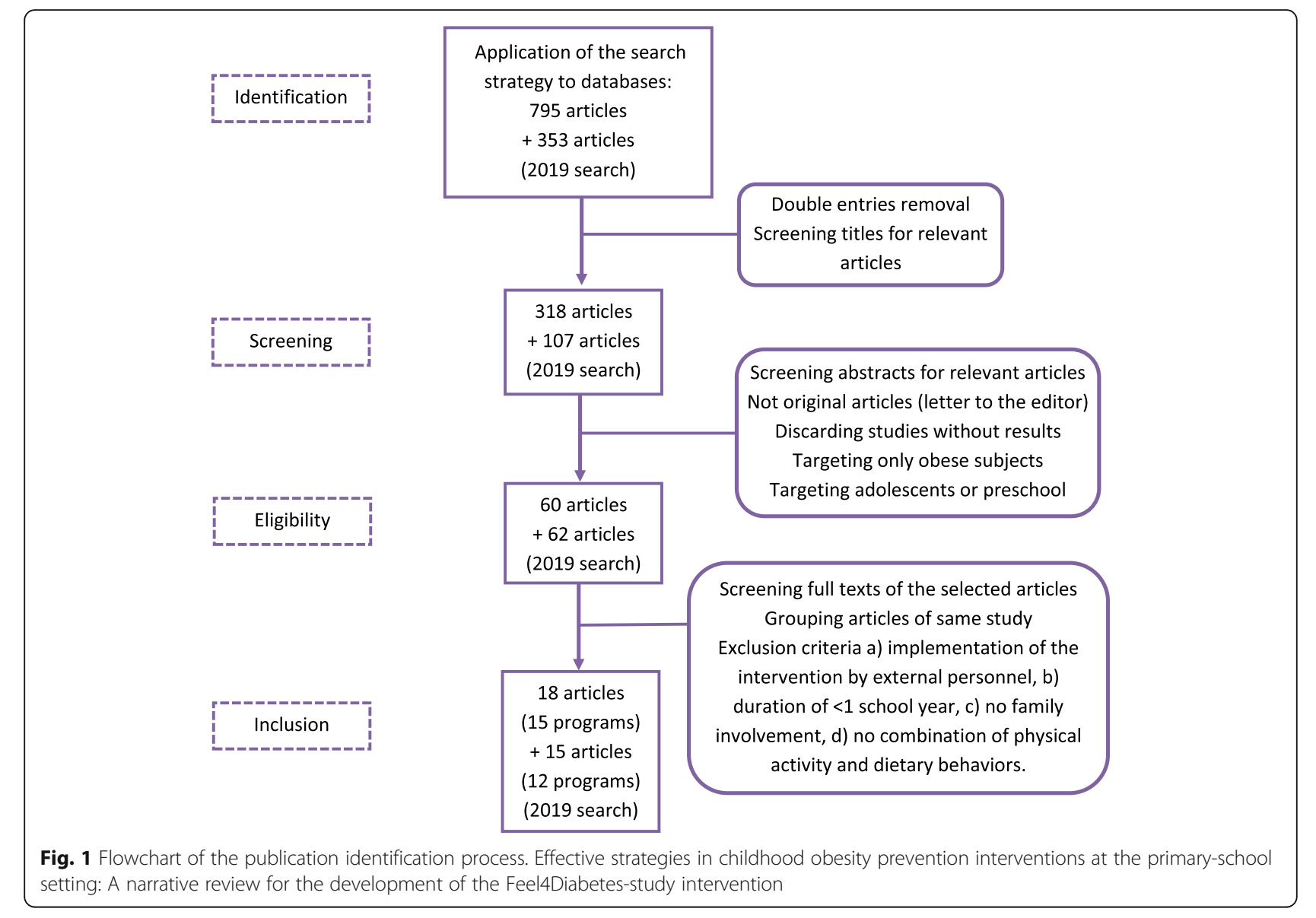

that remained, full-texts were extracted and reviewed based on the inclusion/ exclusion criteria. Finally, 27 intervention programs (33 publications) fulfilled the criteria and were included in the current review.

Most of the included interventions $(n=19)$ were implemented in high income countries (HICs), however a few of them $(n=8)$ were specifically focused on vulnerable population groups or low-to-middle income countries (LMICs), while two of the studies implemented in HICs took vulnerable groups under consideration using extra analysis [23]. The sample size varied across the studies, ranging from 108 to 7406 participants.

\section{Outcome and effectiveness of school-based interventions on weight status and energy balance related behaviors}

Out of the 27 intervention studies (33 publications) included in the current review, 15 (55\% of included studies) presented significant effect on weight status and/ or overweight/ obesity or clinical indices, 3 ( $11 \%$ of included studies) presented significant effect on most target behaviors while 9 (33\% of included studies) presented significant effect on some/few target behaviors. Only a few interventions $(n=4)$ followed-up the participants post-intervention, showing promising longterm effectiveness especially in high-socioeconomic status (SES) groups.

\section{Intervention strategies}

Key strategies have been identified from the effective intervention programs. In studies including various intervention strategies and several outcomes, no direct cause-effect link could be identified, however the strategies or the combination of strategies used in successful interventions were found. Regarding teachers' involvement, having the teachers trained by health professionals to deliver the intervention, coordinate school-based activities and promote healthy energy-balance related behaviors (EBRBs) during school hours instead of having researchers or other personnel implementing the intervention has been suggested as an effective strategy. Inclass material (workbooks, posters, manuals) was used to facilitate the process. Moreover, teachers acting as rolemodels and getting actively involved in all targeted EBRBs has been highlighted as another effective strategy.

Regarding school policies, several strategies such as the increased water accessibility, the free provision of 
Table 1 Overview of the publications included in the review

\begin{tabular}{|c|c|c|c|c|c|c|c|c|}
\hline Publication & $\begin{array}{l}\text { Program } \\
\text { name }\end{array}$ & Country & $\begin{array}{l}\text { Population } \\
\text { group }\end{array}$ & Setting & $\begin{array}{l}\text { Intervention } \\
\text { duration }\end{array}$ & $\begin{array}{l}\text { Intervention } \\
\text { Approach \& } \\
\text { Theoretical } \\
\text { framework }\end{array}$ & $\begin{array}{l}\text { Post-trial } \\
\text { follow-up } \\
\text { duration }\end{array}$ & $\begin{array}{l}\text { Outcomes \& } \\
\text { Effectiveness score }\end{array}$ \\
\hline $\begin{array}{l}\text { Angelopoulos } \\
\text { et al., } 2009 \\
\text { [19] }\end{array}$ & $\begin{array}{l}\text { CHILDREN } \\
\text { study }\end{array}$ & Greece & $\begin{array}{l}13 \text { schools } \\
646 \text { pupils } \\
5 \text { th grade } \\
\text { (Mean } \pm \text { SD: } \\
10.3 \pm 0.4 \\
\text { years) }\end{array}$ & - & $\begin{array}{l}1 \text { school } \\
\text { year }\end{array}$ & $\begin{array}{l}\text { School and home } \\
\text { environment } \\
\text { (availability of } \\
\text { healthy choices) } \\
\text { Policies (Children's } \\
\text { and teachers in- } \\
\text { class-material, En- } \\
\text { joyable fitness clas- } \\
\text { ses, Schoolyards } \\
\text { open after school } \\
\text { hours) } \\
\text { Parents } \\
\text { engagement } \\
\text { (Meetings to } \\
\text { support children's } \\
\text { energy-balance re- } \\
\text { lated behaviors } \\
\text { (EBRBs), Increasing } \\
\text { parental support, } \\
\text { Overcoming the } \\
\text { barriers in acces- } \\
\text { sing physical activ- } \\
\text { ity (PA) areas)) } \\
\text { Teachers acting as } \\
\text { role-models } \\
\text { Theory of Planned } \\
\text { Behavior }\end{array}$ & - & $\begin{array}{l}\text { Significant } \\
\text { favorable effect on } \\
\text { fruits consumption } \\
\text { and fats/oils and } \\
\text { sweets/beverages } \\
\text { consumption. } \\
\text { Significant } \\
\text { favorable effect on } \\
\text { BMI ( } P=0.047) \\
\text { could be explained } \\
\text { by the changes in } \\
\text { fruit and fats/oils } \\
\text { intake. } \\
(+++)\end{array}$ \\
\hline $\begin{array}{l}\text { Benjamins } \\
\text { et al., } 2010 \\
\text { [20] }\end{array}$ & - & Chicago & $\begin{array}{l}2 \text { schools } \\
581 \text { students } \\
1 \text { st-8th grade }\end{array}$ & Jewish schools & $\begin{array}{l}2 \text { school } \\
\text { years }\end{array}$ & $\begin{array}{l}\text { Formation of a } \\
\text { wellness council } \\
\text { Writing of a } \\
\text { wellness } \\
\text { policyHealth } \\
\text { education (via } \\
\text { health lessons) } \\
\text { Physical education } \\
\text { (by providing } \\
\text { funding to increase } \\
\text { PA, gender } \\
\text { separated } \\
\text { swimming lessons) } \\
\text { School } \\
\text { environment } \\
\text { (removing vending } \\
\text { machines, healthy } \\
\text { lunches, skim-milk } \\
\text { promotion, fruit of } \\
\text { the week program) } \\
\text { Family involvement } \\
\text { (school events, } \\
\text { meetings, } \\
\text { newsletters) } \\
\text { Staff wellness (role- } \\
\text { models, private } \\
\text { dietary } \\
\text { consultations, } \\
\text { educational } \\
\text { sessions) } \\
\text { The Centers for } \\
\text { Disease Control } \\
\text { (CDC) ecological } \\
\text { model-based Co- } \\
\text { ordinated School } \\
\text { Health Program }\end{array}$ & - & $\begin{array}{l}\text { Significant } \\
\text { favorable effect n } \\
\text { the percentage of } \\
\text { older students } \\
\text { regularly meeting } \\
\text { physical activity } \\
\text { guidelines. Few } \\
\text { changes in } \\
\text { attitudes, other } \\
\text { behaviors, or } \\
\text { environmental } \\
\text { factors were seen. } \\
(++)\end{array}$ \\
\hline
\end{tabular}


Table 1 Overview of the publications included in the review (Continued)

\begin{tabular}{|c|c|c|c|c|c|c|c|c|}
\hline Publication & $\begin{array}{l}\text { Program } \\
\text { name }\end{array}$ & Country & $\begin{array}{l}\text { Population } \\
\text { group }\end{array}$ & Setting & $\begin{array}{l}\text { Intervention } \\
\text { duration }\end{array}$ & $\begin{array}{l}\text { Intervention } \\
\text { Approach \& } \\
\text { Theoretical } \\
\text { framework }\end{array}$ & $\begin{array}{l}\text { Post-trial } \\
\text { follow-up } \\
\text { duration }\end{array}$ & $\begin{array}{l}\text { Outcomes \& } \\
\text { Effectiveness score }\end{array}$ \\
\hline $\begin{array}{l}\text { Bhave et al.., } \\
2017 \text { [21] }^{\text {a }}\end{array}$ & $\begin{array}{l}\text { SYM-KEM } \\
\text { Study }\end{array}$ & India & $\begin{array}{l}865 \text { children } \\
\text { 3rd and 4th } \\
\text { grades (age } \\
\text { range: } 7.7- \\
\text { 9.6 years). }\end{array}$ & $\begin{array}{l}\text { Academically } \\
\text { competitive } \\
\text { Indian school }\end{array}$ & $\begin{array}{l}2 \text { school } \\
\text { years }\end{array}$ & $\begin{array}{l}\text { Increased extra- } \\
\text { and intra-curricular } \\
\text { physical activity } \\
\text { sessions } \\
\text { Daily yoga-based } \\
\text { breathing exercises } \\
\text { Making physical } \\
\text { activity a 'scoring' } \\
\text { subject } \\
\text { Nutrition education } \\
\text { Healthier school } \\
\text { meals } \\
\text { Removal of fast- } \\
\text { food hawkers from } \\
\text { the school environs } \\
\text { Health and } \\
\text { nutrition education } \\
\text { for teachers, pupils } \\
\text { and families } \\
\text { Theory Framework } \\
\text { not mentioned }\end{array}$ & 5 years & $\begin{array}{l}\text { After five years the } \\
\text { intervention had } \\
\text { significant } \\
\text { favorable effect on } \\
\text { running, long- } \\
\text { jump, sit-up and } \\
\text { push-up tests. } \\
\text { Significant } \\
\text { favorable effect on } \\
\text { sedentary time } \\
\text { (watching TV and } \\
\text { studying), active } \\
\text { play time and fruit } \\
\text { consumption. } \\
\text { Significant } \\
\text { favorable effect on } \\
\text { waist } \\
\text { circumference. } \\
\text { No effect on BMl } \\
\text { or the prevalence } \\
\text { of overweight/ } \\
\text { obesity. } \\
\text { (+++) }\end{array}$ \\
\hline $\begin{array}{l}\text { Brandstetter } \\
\text { et al., } 2012 \\
\text { [22] }\end{array}$ & URMEL-ICE & Germany & $\begin{array}{l}945 \text { children } \\
\text { 2nd grade } \\
\text { (Mean } \pm \text { SD: } \\
7.6 \pm 0.4 \\
\text { years) }\end{array}$ & - & $\begin{array}{l}1 \text { school } \\
\text { year }\end{array}$ & $\begin{array}{l}29 \text { teaching } \\
\text { modules, } \\
2 \text { short exercise } \\
\text { blocks per day } \\
6 \text { family homework } \\
\text { lessons } \\
\text { Parental material } \\
\text { Teachers training } \\
\text { Social Cognitive } \\
\text { theory }\end{array}$ & - & $\begin{array}{l}\text { No statistically } \\
\text { significant effect of } \\
\text { the intervention on } \\
\text { BMl. } \\
\text { Significant favorable } \\
\text { effect on waist } \\
\text { circumference and } \\
\text { subscapular skinfold } \\
\text { thickness before } \\
\text { additional } \\
\text { adjustment for } \\
\text { individual time lag } \\
\text { between baseline } \\
\text { and follow-up. } \\
\text { The intervention } \\
\text { group revealed a } \\
\text { higher percentage } \\
\text { of children with an } \\
\text { improvement and } \\
\text { a lower percentage } \\
\text { with a worsening } \\
\text { of the health- } \\
\text { relevant behaviors } \\
\text { compared to the } \\
\text { control group. } \\
\text { (+++) }\end{array}$ \\
\hline $\begin{array}{l}\text { Cohen et al., } \\
2014 \text { [23] }\end{array}$ & $\begin{array}{l}\text { The CHANGE } \\
\text { study }\end{array}$ & $\begin{array}{l}\text { California, } \\
\text { Kentucky, } \\
\text { Mississippi } \\
\text { and South } \\
\text { Carolina, USA }\end{array}$ & $\begin{array}{l}8 \text { schools432 } \\
\text { children } \\
\text { grades 1-6th } \\
\text { (Mean } \pm \text { SD: } \\
8.6 \pm 1.5 \\
\text { years) }\end{array}$ & $\begin{array}{l}\text { rural low-SES } \\
\text { areas }\end{array}$ & $\begin{array}{l}2 \text { school } \\
\text { years }\end{array}$ & $\begin{array}{l}\text { Food service } \\
\text { component } \\
\text { (healthier choices) } \\
\text { Educational } \\
\text { curriculum every } \\
\text { week } \\
\text { Parent and } \\
\text { community } \\
\text { outreach } \\
\text { components } \\
\text { throughout the } \\
\text { school district to } \\
\text { promote the } \\
\text { healthy lifestyle } \\
\text { changes }\end{array}$ & - & $\begin{array}{l}\text { Significant } \\
\text { favorable effect on } \\
\text { vegetables } \\
\text { consumption and } \\
\text { on combined fruits } \\
\text { and vegetables } \\
\text { consumption } \\
\text { Favorable trend } \\
\text { toward more fruit } \\
\text { consumption (not } \\
\text { significant) } \\
\text { No significant } \\
\text { effects on whole } \\
\text { grains, legumes, } \\
\text { dairy, potatoes/ }\end{array}$ \\
\hline
\end{tabular}


Table 1 Overview of the publications included in the review (Continued)

\begin{tabular}{|c|c|c|c|c|c|c|c|c|}
\hline Publication & $\begin{array}{l}\text { Program } \\
\text { name }\end{array}$ & Country & $\begin{array}{l}\text { Population } \\
\text { group }\end{array}$ & Setting & $\begin{array}{l}\text { Intervention } \\
\text { duration }\end{array}$ & $\begin{array}{l}\text { Intervention } \\
\text { Approach \& } \\
\text { Theoretical } \\
\text { framework }\end{array}$ & $\begin{array}{l}\text { Post-trial } \\
\text { follow-up } \\
\text { duration }\end{array}$ & $\begin{array}{l}\text { Outcomes \& } \\
\text { Effectiveness score }\end{array}$ \\
\hline & & & & & & $\begin{array}{l}\text { encouraged during } \\
\text { and after the } \\
\text { school day. } \\
\text { Theory Framework } \\
\text { not mentioned }\end{array}$ & & $\begin{array}{l}\text { potato products, } \\
\text { saturated fat, } \\
\text { added sugars, or } \\
\text { dietary fiber } \\
\text { consumption. } \\
(+)\end{array}$ \\
\hline $\begin{array}{l}\text { De Coen } \\
\text { et al., } 2012 \\
\text { [24] }\end{array}$ & POP project & Belgium & $\begin{array}{l}31 \text { schools, } \\
1102 \\
\text { children } \\
3-6 \text { years } \\
\text { (Mean } \pm \text { SD: } \\
5.0 \pm 1.3 \\
\text { years) }\end{array}$ & - & $\begin{array}{l}2 \text { school } \\
\text { years }\end{array}$ & $\begin{array}{l}\text { Family involvement } \\
\text { via educational } \\
\text { strategies, } \\
\text { newsletters, tips, } \\
\text { recipes } \\
\text { Development of an } \\
\text { active playground } \\
\text { Implementation of } \\
\text { health-related } \\
\text { physical education } \\
\text { Environmental and } \\
\text { policy changes to } \\
\text { increase availability } \\
\text { of healthy options } \\
\text { Community } \\
\text { stakeholders, local } \\
\text { policy and media } \\
\text { (meetings, } \\
\text { brochures) } \\
\text { involvement } \\
\text { Socio-ecological } \\
\text { model }\end{array}$ & - & $\begin{array}{l}\text { Significant } \\
\text { favorable effect on } \\
\text { BMI Z-score in the } \\
\text { low-socioeconomic } \\
\text { status (SES) inter- } \\
\text { vention } \\
\text { community. } \\
\text { (+++) only in low- } \\
\text { socioeconomic sta- } \\
\text { tus (SES) groups. }\end{array}$ \\
\hline $\begin{array}{l}\text { Foster et al., } \\
2007 \text { [25] }\end{array}$ & - & $\begin{array}{l}\text { Philadelphia, } \\
\text { USA }\end{array}$ & $\begin{array}{l}10 \text { schools } \\
1349 \\
\text { children } \\
4 \text { th }-6 \text { th } \\
\text { grade } \\
\text { (Mean } \pm S D \text { : } \\
11.1 \pm 1.0 \\
\text { years) }\end{array}$ & - & $\begin{array}{l}2 \text { school } \\
\text { years }\end{array}$ & $\begin{array}{l}\text { School Nutrition } \\
\text { Policy Initiative: } \\
\text { School self- } \\
\text { assessment (school } \\
\text { rating and action } \\
\text { plan for change) } \\
\text { Staff training (10 h) } \\
\text { Nutrition education } \\
\text { (50 h) } \\
\text { Nutrition policy } \\
\text { (cafeteria offering } \\
\text { only healthy } \\
\text { options, removal of } \\
\text { vending machines) } \\
\text { Social marketing } \\
\text { (Slogans, character/ } \\
\text { hero, raffle tickets) } \\
\text { Parent outreach } \\
\text { (meetings, } \\
\text { workshops) } \\
\text { Social marketing } \\
\text { and other theories }\end{array}$ & - & $\begin{array}{l}\text { The intervention } \\
\text { resulted in a } 50 \% \\
\text { reduction in the } \\
\text { incidence of } \\
\text { overweight. } \\
\text { Significantly fewer } \\
\text { children in the } \\
\text { intervention } \\
\text { schools than in the } \\
\text { control schools } \\
\text { became } \\
\text { overweight after } 2 \\
\text { years. The } \\
\text { prevalence of } \\
\text { overweight was } \\
\text { lower in the } \\
\text { intervention } \\
\text { schools. } \\
\text { No differences } \\
\text { were observed in } \\
\text { the incidence or } \\
\text { prevalence of } \\
\text { obesity or in the } \\
\text { remission of } \\
\text { overweight or } \\
\text { obesity at } 2 \text { years. } \\
\text { Significant } \\
\text { favorable effect on } \\
\text { inactive hours per } \\
\text { week and on hours } \\
\text { spent on TV on } \\
\text { weekdays. } \\
\text { (+++) }\end{array}$ \\
\hline $\begin{array}{l}\text { Jensen et al., } \\
2015 \text { [26] }\end{array}$ & $\begin{array}{l}\text { Copenhagen } \\
\text { School Child } \\
\text { Intervention }\end{array}$ & $\begin{array}{l}\text { Copenhagen, } \\
\text { Denmark }\end{array}$ & $\begin{array}{l}18 \text { schools } \\
307 \text { children } \\
\text { with full data }\end{array}$ & - & $\begin{array}{l}3 \text { school } \\
\text { years }\end{array}$ & $\begin{array}{l}\text { Two additional } \\
\text { physical education } \\
\text { (PE) lessons per }\end{array}$ & & $\begin{array}{l}\text { Significant } \\
\text { favorable effect on } \\
\text { dietary fibre intake }\end{array}$ \\
\hline
\end{tabular}


Table 1 Overview of the publications included in the review (Continued)

\begin{tabular}{|c|c|c|c|c|c|c|c|c|}
\hline Publication & $\begin{array}{l}\text { Program } \\
\text { name }\end{array}$ & Country & $\begin{array}{l}\text { Population } \\
\text { group }\end{array}$ & Setting & $\begin{array}{l}\text { Intervention } \\
\text { duration }\end{array}$ & $\begin{array}{l}\text { Intervention } \\
\text { Approach \& } \\
\text { Theoretical } \\
\text { framework }\end{array}$ & $\begin{array}{l}\text { Post-trial } \\
\text { follow-up } \\
\text { duration }\end{array}$ & $\begin{array}{l}\text { Outcomes \& } \\
\text { Effectiveness score }\end{array}$ \\
\hline & $\begin{array}{l}\text { Study } \\
\text { (CoSCIS) }\end{array}$ & & $\begin{array}{l}\text { Mean age } \\
6.8 \text { years at } \\
\text { baseline }\end{array}$ & & & $\begin{array}{l}\text { week } \\
\text { Additional } \\
\text { education of PE } \\
\text { teachers } \\
\text { Improvement of } \\
\text { schoolyard } \\
\text { environment } \\
\text { (recess) } \\
\text { Parent involvement } \\
\text { via newsletters } \\
\text { Establishment of } \\
\text { school canteens } \\
\text { Health education in } \\
\text { the curriculum } \\
\text { Theory Framework } \\
\text { not mentioned }\end{array}$ & & $\begin{array}{l}\text { Favorable trend on } \\
\text { fat and saturated } \\
\text { fatty acids intake. } \\
\text { Significant } \\
\text { favorable effect on } \\
\text { the intake of } \\
\text { saturated fatty } \\
\text { acids among } \\
\text { children of } \\
\text { mothers with } \\
\text { higher education. } \\
(+)\end{array}$ \\
\hline $\begin{array}{l}\text { Kain et al., } \\
2008[27]\end{array}$ & - & Chile & $\begin{array}{l}4 \text { schools } \\
2430 \\
\text { students } \\
\text { 1st-8th grade } \\
\text { (Mean } \pm \text { SD: } \\
10.0 \pm 2.3 \\
\text { years) }\end{array}$ & - & $\begin{array}{l}2 \text { school } \\
\text { years }\end{array}$ & $\begin{array}{l}\text { Teachers' training } \\
\text { Parents' education } \\
\text { via meetings } \\
\text { Children's } \\
\text { educational and PE } \\
\text { classes } \\
\text { Active recess } \\
\text { Theory Framework } \\
\text { not mentioned }\end{array}$ & - & $\begin{array}{l}\text { Significant } \\
\text { favorable effect on } \\
\text { obesity prevalence } \\
\text { and BMI-Z in both } \\
\text { boys and girls } \\
(+++)\end{array}$ \\
\hline $\begin{array}{l}\text { Kesztyues } \\
\text { et al., } 2017 \\
{[28]^{a}}\end{array}$ & $\begin{array}{l}\text { The Baden- } \\
\text { Wurttemberg } \\
\text { Study } \\
\text { "Join the }\end{array}$ & Germany & $\begin{array}{l}1733 \\
\text { children } \\
\text { (Mean } \pm \text { SD: } \\
7.1 \pm 0.6\end{array}$ & - & $\begin{array}{l}1 \text { school } \\
\text { year }\end{array}$ & $\begin{array}{l}\text { Teachers' training } \\
\text { All materials were } \\
\text { integrated into the } \\
\text { regular curriculum }\end{array}$ & - & $\begin{array}{l}\text { Significant } \\
\text { favorable effect on } \\
\text { abdominal obesity } \\
(+++)\end{array}$ \\
\hline $\begin{array}{l}\text { Kobel et al.., } \\
2017 \text { [29] }\end{array}$ & $\begin{array}{l}\text { Healthy Boat" } \\
\text { program }\end{array}$ & & years) & $\begin{array}{l}\text { Migration } \\
\text { background } \\
\text { Sub-sample }\end{array}$ & & $\begin{array}{l}\text { Materials for } \\
\text { children (e.g. } \\
\text { activity breaks) and } \\
\text { for parents (e.g. } \\
\text { family homework } \\
\text { and information } \\
\text { material) } \\
\text { Translation of the } \\
\text { material in order to } \\
\text { also reach parents } \\
\text { with migration } \\
\text { background } \\
\text { Intervention } \\
\text { mapping, social } \\
\text { cognitive theory \& } \\
\text { socio-ecological } \\
\text { model }\end{array}$ & & $\begin{array}{l}\text { Significant } \\
\text { favorable effect on } \\
\text { fruits \& vegetables } \\
\text { intake } \\
\text { Favorable trend on } \\
\text { PA and soft drink } \\
\text { consumption } \\
(+)\end{array}$ \\
\hline $\begin{array}{l}\text { King et al. } \\
2015 \text { [30] }^{\text {a }}\end{array}$ & - & $\begin{array}{l}\text { rural } \\
\text { elementary } \\
\text { schools in the } \\
\text { southern } \\
\text { United States }\end{array}$ & $\begin{array}{l}4 \text { rural } \\
\text { elementary } \\
\text { School } \\
999 \text { children } \\
\text { kindergarten } \\
\text { to third } \\
\text { grade } \\
\text { (Mean age: } \\
7.3 \text { years) }\end{array}$ & $\begin{array}{l}\text { rural, low- } \\
\text { socioeconomic } \\
\text { status } \\
\text { elementary } \\
\text { schools }\end{array}$ & $\begin{array}{l}1 \text { school } \\
\text { year }\end{array}$ & $\begin{array}{l}\text { Nutrition and } \\
\text { health Education } \\
\text { SPARK PE } \\
\text { curriculum } \\
\text { Classroom PA } \\
\text { Strengthening } \\
\text { school wellness } \\
\text { policies } \\
\text { Health promotion } \\
\text { for teachers and } \\
\text { families } \\
\text { Promoting family } \\
\text { involvement and } \\
\text { community } \\
\text { partnerships } \\
\text { Culminating goal } \\
\text { for each school to } \\
\text { achieve 'bronze' or }\end{array}$ & & $\begin{array}{l}\text { Significant } \\
\text { favorable effect on } \\
\text { nutrition and } \\
\text { physical activity } \\
\text { behaviors } \\
\text { Significant } \\
\text { favorable effect on } \\
\text { the percentage of } \\
\text { children meeting } \\
\text { the nutrition } \\
\text { recommendation } \\
\text { Significant } \\
\text { favorable effect on } \\
\text { the percentage of } \\
\text { children meeting } \\
\text { the physical } \\
\text { activity } \\
\text { recommendation }\end{array}$ \\
\hline
\end{tabular}


Table 1 Overview of the publications included in the review (Continued)

\begin{tabular}{|c|c|c|c|c|c|c|c|c|}
\hline Publication & $\begin{array}{l}\text { Program } \\
\text { name }\end{array}$ & Country & $\begin{array}{l}\text { Population } \\
\text { group }\end{array}$ & Setting & $\begin{array}{l}\text { Intervention } \\
\text { duration }\end{array}$ & $\begin{array}{l}\text { Intervention } \\
\text { Approach \& } \\
\text { Theoretical } \\
\text { framework }\end{array}$ & $\begin{array}{l}\text { Post-trial } \\
\text { follow-up } \\
\text { duration }\end{array}$ & $\begin{array}{l}\text { Outcomes \& } \\
\text { Effectiveness score }\end{array}$ \\
\hline & & & & & & $\begin{array}{l}\text { higher status of the } \\
\text { Healthier US School } \\
\text { Challenge } \\
\text { The CDC's } \\
\text { ecological model- } \\
\text { based Coordinated } \\
\text { School Health } \\
\text { Program }\end{array}$ & & $(++)$ \\
\hline $\begin{array}{l}\text { Kipping et al., } \\
2014 \text { [31] }\end{array}$ & AFLY5 & $\begin{array}{l}\text { South west } \\
\text { England }\end{array}$ & $\begin{array}{l}60 \\
\text { schools } 2221 \\
\text { students } \\
\text { 4th grade } \\
\text { (Age range: } \\
\text { 8-9 years) }\end{array}$ & - & $\begin{array}{l}1 \text { school } \\
\text { year }\end{array}$ & $\begin{array}{l}\text { Teacher training } \\
\text { Provision of } 16 \\
\text { lessons \& child- } \\
\text { parent interactive } \\
\text { homework plans } \\
\text { Materials for } \\
\text { lessons and } \\
\text { homework, and } \\
\text { written materials } \\
\text { for school } \\
\text { newsletters and } \\
\text { parents. } \\
\text { Social Cognitive } \\
\text { Theory }\end{array}$ & - & $\begin{array}{l}\text { No effect on the } \\
\text { three primary } \\
\text { outcomes (PA, } \\
\text { sedentary time, } \\
\text { diet) } \\
\text { Significant } \\
\text { favorable effect on } \\
\text { three out of nine } \\
\text { of the secondary } \\
\text { outcomes, i.e. self- } \\
\text { reported time } \\
\text { spent in screen } \\
\text { viewing at the } \\
\text { weekend, self- } \\
\text { reported servings } \\
\text { of snacks per day, } \\
\text { and servings of } \\
\text { high energy drinks } \\
\text { per day. } \\
(+)\end{array}$ \\
\hline $\begin{array}{l}\text { Lawlor et al., } \\
2016 \text { [32] }^{\mathrm{a}}\end{array}$ & & & & - & & & - & $\begin{array}{l}\text { Significant } \\
\text { favorable effect on } \\
3 / 10 \text { potential } \\
\text { mediators, i.e. fruit } \\
\text { and vegetable self- } \\
\text { efficacy; child- } \\
\text { reported maternal } \\
\text { limitation of seden- } \\
\text { tary behavior; and } \\
\text { knowledge. } \\
(+)\end{array}$ \\
\hline $\begin{array}{l}\text { Anderson } \\
\text { et al., } 2016 \\
\text { [33] }^{\mathrm{a}}\end{array}$ & & & & - & & & $\begin{array}{l}1 \text { year post } \\
\text { intervention }\end{array}$ & $\begin{array}{l}\text { No effect on mean } \\
\text { child-reported } \\
\text { screen viewing at } \\
\text { the weekend, serv- } \\
\text { ings of snacks per } \\
\text { day, servings of } \\
\text { high-energy drinks } \\
\text { per day, servings of } \\
\text { high-fat foods per } \\
\text { day. } \\
(-)\end{array}$ \\
\hline $\begin{array}{l}\text { Llargues et al., } \\
2011 \text { [15] }\end{array}$ & AVall study & $\begin{array}{l}\text { Granollers } \\
\text { Spain }\end{array}$ & $\begin{array}{l}16 \\
\text { schools500 } \\
\text { students } \\
1 \text { st grade } \\
\text { (Age range: } \\
5-6 \text { years) }\end{array}$ & - & $\begin{array}{l}2 \text { school } \\
\text { years }\end{array}$ & $\begin{array}{l}\text { Educational } \\
\text { sessions to } \\
\text { promote healthy } \\
\text { eating habits and } \\
\text { physical activity } \\
\text { Information session } \\
\text { with the parents } \\
\text { Distribution of } \\
\text { healthy recipes } \\
\text { Research, Vision, } \\
\text { Action and } \\
\text { Change (IVAC) } \\
\text { methodology }\end{array}$ & - & $\begin{array}{l}\text { Significant favorable } \\
\text { effect on BMl and } \\
\text { the prevalence of } \\
\text { overweight children } \\
\text { Significant favorable } \\
\text { effect on the } \\
\text { proportion of } \\
\text { children that ate a } \\
\text { second piece of fruit } \\
\text { Significant favorable } \\
\text { effect on the } \\
\text { consumption of fish. } \\
(+++)\end{array}$ \\
\hline
\end{tabular}


Table 1 Overview of the publications included in the review (Continued)

\begin{tabular}{|c|c|c|c|c|c|c|c|c|}
\hline Publication & $\begin{array}{l}\text { Program } \\
\text { name }\end{array}$ & Country & $\begin{array}{l}\text { Population } \\
\text { group }\end{array}$ & Setting & $\begin{array}{l}\text { Intervention } \\
\text { duration }\end{array}$ & $\begin{array}{l}\text { Intervention } \\
\text { Approach \& } \\
\text { Theoretical } \\
\text { framework }\end{array}$ & $\begin{array}{l}\text { Post-trial } \\
\text { follow-up } \\
\text { duration }\end{array}$ & $\begin{array}{l}\text { Outcomes \& } \\
\text { Effectiveness score }\end{array}$ \\
\hline $\begin{array}{l}\text { Lloyd et al., } \\
2017 \text { [34] }\end{array}$ & $\begin{array}{l}\text { Healthy } \\
\text { Lifestyles } \\
\text { Programme } \\
\text { (HeLP) }\end{array}$ & $\begin{array}{l}\text { South West } \\
\text { of England }\end{array}$ & $\begin{array}{l}32 \text { schools } \\
1324 \\
\text { children } \\
\text { (Age range: } \\
\text { 9-10 years) }\end{array}$ & - & $\begin{array}{l}1 \text { school } \\
\text { year }\end{array}$ & $\begin{array}{l}\text { Dynamic \& } \\
\text { interactive activities } \\
\text { e.g. } \\
\text { Physical activity } \\
\text { workshops } \\
\text { Education sessions } \\
\text { delivered by } \\
\text { teachers with short } \\
\text { homework tasks } \\
\text { Drama sessions } \\
\text { Goal setting to } \\
\text { modify behavior } \\
\text { with parental } \\
\text { support and one- } \\
\text { to-one discussions } \\
\text { with the project's } \\
\text { coordinators } \\
\text { Extensive } \\
\text { stakeholder } \\
\text { involvement } \\
\text { Intervention } \\
\text { mapping }\end{array}$ & - & $\begin{array}{l}\text { No significant } \\
\text { effects on } \\
\text { anthropometric or } \\
\text { physical activity } \\
\text { outcomes } \\
\text { Significant } \\
\text { favorable effect on } \\
\text { the adjusted } \\
\text { means of the Food } \\
\text { Intake } \\
\text { Questionnaire } \\
\text { scores (both } \\
\text { weekly and } \\
\text { weekday) for } \\
\text { energy-dense } \\
\text { snacks and nega- } \\
\text { tive food markers } \\
\text { (+) }\end{array}$ \\
\hline $\begin{array}{l}\text { Manios et al., } \\
2002[35]\end{array}$ & - & Crete, Greece & $\begin{array}{l}40 \text { schools } \\
5681 \text { students } \\
\text { 1st grade } \\
\text { (Age range: } \\
6-7 \text { years) }\end{array}$ & - & $\begin{array}{l}6 \text { school } \\
\text { years }\end{array}$ & $\begin{array}{l}\text { Multicomponent } \\
\text { workbooks for } \\
\text { students } \\
\text { Teaching aids } \\
\text { included posters, } \\
\text { audio-taped fairy } \\
\text { tales for classroom } \\
\text { use, workbooks, } \\
\text { and teaching man- } \\
\text { uals Non- } \\
\text { competitive } \\
\text { activities } \\
\text { Meetings with } \\
\text { parents } \\
\text { Booklet distribution }\end{array}$ & & $\begin{array}{l}\text { Significant } \\
\text { favorable effect on } \\
\text { biceps skinfold } \\
\text { Significant } \\
\text { favorable effect on } \\
\text { total energy intake, } \\
\text { consumption of } \\
\text { total fat and } \\
\text { saturated fat } \\
\text { Significant } \\
\text { favorable effect on } \\
\text { time devoted to } \\
\text { leisure time } \\
\text { physical activity } \\
(+++)\end{array}$ \\
\hline $\begin{array}{l}\text { Manios et al., } \\
2006 \text { [36] }\end{array}$ & & & & & & $\begin{array}{l}\text { Social Cognitive } \\
\text { theory }\end{array}$ & $\begin{array}{l}4 \text { years post } \\
\text { intervention }\end{array}$ & $\begin{array}{l}\text { Significant } \\
\text { favorable effect on } \\
\text { MVPA levels for } \\
\text { males and on } \\
\text { males meeting the } \\
\text { recommendations } \\
\text { for physical activity } \\
(+)\end{array}$ \\
\hline $\begin{array}{l}\text { Manios et al., } \\
2006 \text { [37] }\end{array}$ & & & & & & & & $\begin{array}{l}\text { Significant } \\
\text { favorable effect on } \\
\text { total cholesterol, } \\
\text { LDL-cholesterol, } \\
\text { HDL-cholesterol } \\
\text { and total choles- } \\
\text { terol: HDL- } \\
\text { cholestrol ratio } \\
\text { Significant } \\
\text { favorable effect on } \\
\text { leisure-time phys- } \\
\text { ical activities and } \\
\text { BMl } \\
\text { No effects on } \\
\text { fitness and dietary } \\
\text { indices examined. } \\
\text { (+++) }\end{array}$ \\
\hline
\end{tabular}


Table 1 Overview of the publications included in the review (Continued)

\begin{tabular}{|c|c|c|c|c|c|c|c|c|}
\hline Publication & $\begin{array}{l}\text { Program } \\
\text { name }\end{array}$ & Country & $\begin{array}{l}\text { Population } \\
\text { group }\end{array}$ & Setting & $\begin{array}{l}\text { Intervention } \\
\text { duration }\end{array}$ & $\begin{array}{l}\text { Intervention } \\
\text { Approach \& } \\
\text { Theoretical } \\
\text { framework }\end{array}$ & $\begin{array}{l}\text { Post-trial } \\
\text { follow-up } \\
\text { duration }\end{array}$ & $\begin{array}{l}\text { Outcomes \& } \\
\text { Effectiveness score }\end{array}$ \\
\hline $\begin{array}{l}\text { Mårild et al., } \\
2015[38]^{a}\end{array}$ & IDEFICS study & $\begin{array}{l}\text { Belgium, } \\
\text { Cyprus, } \\
\text { Estonia, } \\
\text { Germany, } \\
\text { Hungary, } \\
\text { Italy, } \\
\text { Spain, } \\
\text { Sweden }\end{array}$ & $\begin{array}{l}7406 \\
\text { children (age } \\
2-9.9 \text { years) } \\
\text { of the } 16,228 \\
\text { participating }\end{array}$ & - & $\begin{array}{l}1 \text { school } \\
\text { year }\end{array}$ & $\begin{array}{l}\text { Sustainable change } \\
\text { in health behaviors } \\
\text { and in the } \\
\text { community } \\
\text { environment in } \\
\text { cooperation with } \\
\text { political leaders, } \\
\text { teachers and } \\
\text { stakeholders. } \\
\text { Intervention } \\
\text { Mapping }\end{array}$ & - & $\begin{array}{l}\text { No effects on } \\
\text { insulin, HOMA-IR, } \\
\text { CRP or the MetS } \\
\text { score } \\
\text { Significant } \\
\text { favorable effect on } \\
\text { fasting glucose, a } \\
\text { pattern driven by } \\
\text { three of the eight } \\
\text { countries and } \\
\text { more pronounced } \\
\text { in children of } \\
\text { parents with low } \\
\text { education. } \\
\text { Significant } \\
\text { unfavorable effect } \\
\text { on HbA1c and } \\
\text { waist } \\
\text { circumference } \\
\text { increased more } \\
\text { and blood pressure } \\
\text { less in the } \\
\text { intervention } \\
\text { regions. } \\
\text { (+) only in glucose } \\
\text { levels but } \\
\text { otherwise } \\
\text { contradicting } \\
\text { results. }\end{array}$ \\
\hline $\begin{array}{l}\text { Mihrshahi } \\
\text { et al., } 2017 \\
\text { [39] }^{\text {a }}\end{array}$ & $\begin{array}{l}\text { Good Start } \\
\text { Program }\end{array}$ & $\begin{array}{l}\text { Maori and } \\
\text { Pacific } \\
\text { Islander } \\
\text { communities } \\
\text { living in } \\
\text { Queensland }\end{array}$ & $\begin{array}{l}375 \text { children } \\
\text { (6-19 years) }\end{array}$ & $\begin{array}{l}\text { Maori and } \\
\text { Pacific Islander } \\
\text { communities } \\
\text { living in } \\
\text { Queensland }\end{array}$ & $\begin{array}{l}1 \text { school } \\
\text { year }\end{array}$ & $\begin{array}{l}\text { Class activities } \\
\text { focused on one } \\
\text { message each term } \\
\text { related to healthy } \\
\text { eating and physical } \\
\text { activity using } \\
\text { methods such as } \\
\text { cooking sessions } \\
\text { and cultural dance. } \\
\text { Theory Framework } \\
\text { not mentioned }\end{array}$ & - & $\begin{array}{l}\text { Quantitative } \\
\text { uncontrolled pre- } \\
\text { post design. } \\
\text { Significant } \\
\text { favorable time } \\
\text { effect on } \\
\text { knowledge of } \\
\text { correct servings of } \\
\text { fruit and } \\
\text { vegetables, } \\
\text { knowledge of } \\
\text { sugar and caffeine } \\
\text { content of } \\
\text { common sugar- } \\
\text { sweetened drinks, } \\
\text { recognition of the } \\
\text { consequences of } \\
\text { marketing and up- } \\
\text { sizing, and the im- } \\
\text { portance of } \\
\text { controlling portion } \\
\text { size, knowledge of } \\
\text { physical activity } \\
\text { recommendations, } \\
\text { as well as the im- } \\
\text { portance of phys- } \\
\text { ical activity for } \\
\text { preventing heart } \\
\text { disease and im- } \\
\text { proving self- } \\
\text { esteem } \\
\text { Significant } \\
\text { favorable time }\end{array}$ \\
\hline
\end{tabular}


Table 1 Overview of the publications included in the review (Continued)

\begin{tabular}{|c|c|c|c|c|c|c|c|c|}
\hline Publication & $\begin{array}{l}\text { Program } \\
\text { name }\end{array}$ & Country & $\begin{array}{l}\text { Population } \\
\text { group }\end{array}$ & Setting & $\begin{array}{l}\text { Intervention } \\
\text { duration }\end{array}$ & $\begin{array}{l}\text { Intervention } \\
\text { Approach \& } \\
\text { Theoretical } \\
\text { framework }\end{array}$ & $\begin{array}{l}\text { Post-trial } \\
\text { follow-up } \\
\text { duration }\end{array}$ & $\begin{array}{l}\text { Outcomes \& } \\
\text { Effectiveness score }\end{array}$ \\
\hline & & & & & & & & $\begin{array}{l}\text { effect on some } \\
\text { attitudes to } \\
\text { vegetables and } \\
\text { sugar-sweetened } \\
\text { drinks and the re- } \\
\text { ported intake of } \\
\text { vegetables } \\
(+)\end{array}$ \\
\hline $\begin{array}{l}\text { Pablos et al., } \\
2017 \text { [40] }\end{array}$ & $\begin{array}{l}\text { Healthy } \\
\text { Habits } \\
\text { Program } \\
(\text { HHP) }\end{array}$ & $\begin{array}{l}\text { Valencian } \\
\text { Community, } \\
\text { Spain }\end{array}$ & $\begin{array}{l}2 \text { schools } \\
158 \text { children } \\
\text { (10-12 years) }\end{array}$ & - & $\begin{array}{l}1 \text { school } \\
\text { year }\end{array}$ & $\begin{array}{l}\text { Free extracurricular } \\
\text { activity: } \\
\text { brief 10-min talk } \\
\text { about healthy } \\
\text { habits } \\
\text { Physical exercise } \\
\text { session targeting } \\
\text { fun, inclusion and } \\
\text { cooperation, and } \\
\text { safety (themed } \\
\text { games) } \\
\text { All the sessions } \\
\text { were led by the } \\
\text { same specifically- } \\
\text { trained teacher. } \\
\text { A worksheet } \\
\text { designed by the } \\
\text { research team was } \\
\text { given to be } \\
\text { completed at } \\
\text { home ( } 29 \text { in total) } \\
\text { Three } 45 \text {-min talks } \\
\text { for parents and } \\
\text { teachers about } \\
\text { healthy habits for } \\
\text { school children. } \\
\text { Theory Framework } \\
\text { not mentioned }\end{array}$ & & $\begin{array}{l}\text { Significant } \\
\text { favorable effect on } \\
\text { triglycerides, blood } \\
\text { glucose and } \\
\text { VO2max, breakfast } \\
\text { habits and quality } \\
\text { of diet, the } \\
\text { prevalence of } \\
\text { normal levels for } \\
\text { total cholesterol, } \\
\text { blood pressure and } \\
\text { BMl. } \\
(+++)\end{array}$ \\
\hline $\begin{array}{l}\text { Plachta- } \\
\text { Danielzik } \\
\text { et al., } 2007 \\
\text { [41] }\end{array}$ & KOPS study & Kiel, Germany & $\begin{array}{l}32 \\
\text { schools } 1764 \\
\text { students } \\
\text { (Age: } 6 \text { years) }\end{array}$ & - & $\begin{array}{l}1 \text { school } \\
\text { year }\end{array}$ & $\begin{array}{l}\text { Teachers training on } \\
\text { a structured nutrition } \\
\text { education program. } \\
\text { Health messages } \\
\text { were given to } \\
\text { children, parents, } \\
\text { and teachers, } \\
\text { conveyed as } \\
\text { nutrition fairy tales, } \\
\text { interactive games, } \\
\text { and by preparing a } \\
\text { healthy breakfast. } \\
\text { Six nutrition units } \\
\text { performed during } 2 \\
\text { to } 3 \text { weeks within } \\
\text { the second term of } \\
\text { the first school }\end{array}$ & $\begin{array}{l}4 \text { years post } \\
\text { intervention }\end{array}$ & $\begin{array}{l}\text { No effect on mean } \\
\text { BMl } \\
\text { Significant } \\
\text { favorable effect on } \\
\text { the prevalence of } \\
\text { overweight and } \\
\text { obesity in children } \\
\text { from families with } \\
\text { high } \\
\text { socioeconomic } \\
\text { status and } \\
\text { marginally } \\
\text { significant in } \\
\text { children of normal- } \\
\text { weight mothers. } \\
\text { (+++) only in high- } \\
\text { SES groups. }\end{array}$ \\
\hline $\begin{array}{l}\text { Plachta- } \\
\text { Danielzik } \\
\text { et al., } 2011 \\
\text { [42] }\end{array}$ & & & & & & $\begin{array}{l}\text { year. After each } \\
\text { unit, running } \\
\text { games were } \\
\text { offered for } 20 \text { min } \\
\text { on the } \\
\text { schoolyard.Parents } \\
\text { were informed } \\
\text { during a parental } \\
\text { school meeting. } \\
\text { Theory Framework } \\
\text { not mentioned }\end{array}$ & $\begin{array}{l}8 \text { years post } \\
\text { intervention }\end{array}$ & $\begin{array}{l}\text { No effect on mean } \\
\text { BMI, lifestyle and } \\
\text { blood pressure } \\
\text { Significant favorable } \\
\text { effect on the 8-year } \\
\text { change in BMl- } \\
\text { standard deviation } \\
\text { scores (SDS) in high } \\
\text { SES groups } \\
\text { (+++) only in high- } \\
\text { SES groups. }\end{array}$ \\
\hline
\end{tabular}


Table 1 Overview of the publications included in the review (Continued)

\begin{tabular}{|c|c|c|c|c|c|c|c|c|}
\hline Publication & $\begin{array}{l}\text { Program } \\
\text { name }\end{array}$ & Country & $\begin{array}{l}\text { Population } \\
\text { group }\end{array}$ & Setting & $\begin{array}{l}\text { Intervention } \\
\text { duration }\end{array}$ & $\begin{array}{l}\text { Intervention } \\
\text { Approach \& } \\
\text { Theoretical } \\
\text { framework }\end{array}$ & $\begin{array}{l}\text { Post-trial } \\
\text { follow-up } \\
\text { duration }\end{array}$ & $\begin{array}{l}\text { Outcomes \& } \\
\text { Effectiveness score }\end{array}$ \\
\hline $\begin{array}{l}\text { Rush et al., } \\
2014 \text { [43] }^{a}\end{array}$ & $\begin{array}{l}\text { Project } \\
\text { Energize }\end{array}$ & New Zealand & $\begin{array}{l}193 \text { primary } \\
\text { schools } \\
4804 \\
\text { children } \\
\text { (Age range: } \\
\text { 6-11 years) }\end{array}$ & $\begin{array}{l}42 \% \text { Mãori, the } \\
\text { indigenous } \\
\text { people of New } \\
\text { Zealand }\end{array}$ & & $\begin{array}{l}\text { Encourage healthy } \\
\text { behaviors daily } \\
\text { Healthy choices } \\
\text { availability and } \\
\text { decrease the } \\
\text { availability of high } \\
\text { energy/ low } \\
\text { nutrient foods } \\
\text { Increase the } \\
\text { awareness of } \\
\text { healthy choices } \\
\text { Consistent nutrition } \\
\text { messages in all } \\
\text { aspects of school } \\
\text { and community } \\
\text { interaction e.g., } \\
\text { healthy fundraising } \\
\text { options } \\
\text { Encourage } \\
\text { lunchtime physical } \\
\text { activity at least } \\
\text { twice a week } \\
\text { Raise awareness of } \\
\text { incidental activity } \\
\text { opportunities at } \\
\text { home and school } \\
\text { Theory Framework } \\
\text { not mentioned }\end{array}$ & & $\begin{array}{l}\text { Significant } \\
\text { favorable effect on } \\
\text { the combined } \\
\text { prevalence of } \\
\text { obesity and } \\
\text { overweight and } \\
\text { BMl, physical } \\
\text { fitness in both } \\
\text { boys and girls, } \\
\text { both indigenous } \\
\text { Maori and non- } \\
\text { Maori children, ano } \\
\text { across SES. } \\
(+++)\end{array}$ \\
\hline $\begin{array}{l}\text { Sacchetti } \\
\text { et al., } 2015 \\
{[44]^{\mathrm{a}}}\end{array}$ & $\begin{array}{l}\text { SAMBA } \\
\text { project }\end{array}$ & Bologna, Italy & $\begin{array}{l}11 \text { classes } \\
234 \text { school } \\
\text { children } \\
\text { (Age range: } \\
8-11 \text { years) }\end{array}$ & - & $\begin{array}{l}3 \text { school } \\
\text { years }\end{array}$ & $\begin{array}{l}\text { Training modules } \\
\text { for teachers } \\
\text { Training modules } \\
\text { for instructors of } \\
\text { local sport societies } \\
\text { Educational } \\
\text { activities in class } \\
\text { Free and structured } \\
\text { games inside the } \\
\text { school and in the } \\
\text { open. } \\
\text { Cookery workshops } \\
\text { and sensory } \\
\text { courses for parents } \\
\text { and teachers. } \\
\text { Moments of } \\
\text { movement with } \\
\text { parents in free } \\
\text { time: homework } \\
\text { Dog walking \& } \\
\text { Home-school } \\
\text { routes on foot/by } \\
\text { bike } \\
\text { Creation of didactic } \\
\text { materials (recipes, } \\
\text { leaflets, DVDs, food } \\
\text { pyramids) } \\
\text { Precede-Proceed } \\
\text { Model }\end{array}$ & & $\begin{array}{l}\text { Significant } \\
\text { favorable effect on } \\
\text { weight, the } \\
\text { percentage of } \\
\text { children who } \\
\text { consumed an } \\
\text { adequate mid- } \\
\text { morning snack, the } \\
\text { percentage of chil- } \\
\text { dren who con- } \\
\text { sumed snacks and } \\
\text { drinks after the } \\
\text { dinner and the } \\
\text { percentage of } \\
\text { those who con- } \\
\text { sumed five or } \\
\text { more portions of } \\
\text { fruits and vegeta- } \\
\text { bles daily. } \\
(+++)\end{array}$ \\
\hline $\begin{array}{l}\text { Sahota et al., } \\
2001[45]\end{array}$ & APPLES & Leeds, UK & $\begin{array}{l}10 \text { schools } \\
634 \text { students } \\
\text { (Age range: } \\
7-11 \text { years) }\end{array}$ & - & $\begin{array}{l}1 \text { school } \\
\text { year }\end{array}$ & $\begin{array}{l}\text { Teacher training } \\
\text { Modification of } \\
\text { school meals } \\
\text { Development of } \\
\text { school action plans } \\
\text { (Individualized on }\end{array}$ & - & $\begin{array}{l}\text { Significant } \\
\text { favorable effect on } \\
\text { vegetable } \\
\text { consumption } \\
\text { No effect on } \\
\text { physical activity or }\end{array}$ \\
\hline
\end{tabular}


Table 1 Overview of the publications included in the review (Continued)

\begin{tabular}{|c|c|c|c|c|c|c|c|c|}
\hline Publication & $\begin{array}{l}\text { Program } \\
\text { name }\end{array}$ & Country & $\begin{array}{l}\text { Population } \\
\text { group }\end{array}$ & Setting & $\begin{array}{l}\text { Intervention } \\
\text { duration }\end{array}$ & $\begin{array}{l}\text { Intervention } \\
\text { Approach \& } \\
\text { Theoretical } \\
\text { framework }\end{array}$ & $\begin{array}{l}\text { Post-trial } \\
\text { follow-up } \\
\text { duration }\end{array}$ & $\begin{array}{l}\text { Outcomes \& } \\
\text { Effectiveness score }\end{array}$ \\
\hline & & & & & & $\begin{array}{l}\text { school level based } \\
\text { on their needs) } \\
\text { Parental } \\
\text { involvement } \\
\text { Theory Framework } \\
\text { not mentioned }\end{array}$ & & $\begin{array}{l}\text { sedentary behavior } \\
(+)\end{array}$ \\
\hline $\begin{array}{l}\text { Shofan et al., } \\
2011 \text { [46] }\end{array}$ & - & Israel & $\begin{array}{l}2 \text { schools } \\
108 \text { students } \\
\text { 4th-6th } \\
\text { grade } \\
\text { (Age range: } \\
\text { 9-11 years) }\end{array}$ & - & $\begin{array}{l}2 \text { school } \\
\text { years }\end{array}$ & $\begin{array}{l}8 \text { nutritional } \\
\text { education } \\
\text { lessonsDouble } \\
\text { physical education } \\
\text { hours (intense } \\
\text { aerobic activity } \\
\text { designed to } \\
\text { increase the } \\
\text { aerobic component } \\
\text { by } 50 \% \text { Regular } \\
\text { parents meetings, } \\
\text { once a month for } \\
\text { one hour per } \\
\text { session for } 10 \\
\text { months a year, } \\
\text { Encouragement of } \\
\text { healthy dietary } \\
\text { habits. } \\
\text { Theory Framework } \\
\text { not mentioned }\end{array}$ & - & $\begin{array}{l}\text { Significant } \\
\text { favorable effect on } \\
\text { the average BMI } \\
\text { percentile } \\
\text { Significant } \\
\text { favorable effect on } \\
\text { weight for boys No } \\
\text { effect on weight or } \\
\text { BMl in girls. } \\
(+++)\end{array}$ \\
\hline $\begin{array}{l}\text { Spiegel et al., } \\
2006[47]^{\mathrm{a}}\end{array}$ & WAY program & USA & $\begin{array}{l}69 \text { classes } \\
1013 \\
\text { students } \\
4 \text { th } \& 5 \text { th } \\
\text { grade } \\
\text { (Age range: } \\
\text { 9-11 years) }\end{array}$ & - & $\begin{array}{l}1 \text { school } \\
\text { year }\end{array}$ & $\begin{array}{l}\text { Intervention } \\
\text { teachers } \\
\text { participated in } \\
\text { workshops on the } \\
\text { intervention and } \\
\text { received program } \\
\text { materials.Family } \\
\text { involvement } \\
\text { through activities } \\
\text { and } \\
\text { discussionsClass } \\
\text { modules } \\
\text { Theory of } \\
\text { Reasoned Action }\end{array}$ & - & $\begin{array}{l}\text { Significant } \\
\text { favorable effect on } \\
\text { BMI, the } \\
\text { consumption of } \\
\text { fruits and } \\
\text { vegetables and } \\
\text { physical activity } \\
\text { levels } \\
(+++)\end{array}$ \\
\hline $\begin{array}{l}\text { Springer et al., } \\
2012 \text { [48] }\end{array}$ & $\begin{array}{l}\text { Marathon } \\
\text { Kids }\end{array}$ & Texas, USA & $\begin{array}{l}511 \\
\text { students4th- } \\
\text { 5th grade } \\
\text { Mean age: } \\
10 \text { years }\end{array}$ & Low-SES area & $\begin{array}{l}1 \text { school } \\
\text { year }\end{array}$ & $\begin{array}{l}\text { Miles } \\
\text { trackingNumber of } \\
\text { F\&V tracking } \\
\text { Kick-off and Final } \\
\text { Mile Run in public } \\
\text { venues with } \\
\text { celebrities-mayors- } \\
\text { professional ath- } \\
\text { letes as hosts/ pub- } \\
\text { lic role- } \\
\text { modelsCommunity } \\
\text { events/ festivalsT- } \\
\text { shirts-medals at the } \\
\text { end, logo, stickers, } \\
\text { advertising on } \\
\text { buses \& scheduled } \\
\text { time for walking/ } \\
\text { running at school } \\
\text { Ecological Model } \\
\text { by Sallis et al, } \\
2006\end{array}$ & - & $\begin{array}{l}\text { Significant } \\
\text { favorable effect on } \\
\text { the mean time of } \\
\text { running in past } 7 \\
\text { days the mean fruit } \\
\text { and vegetable } \\
\text { consumption, } \\
\text { athletic identity } \\
\text { self-concept, PA } \\
\text { outcome expecta- } \\
\text { tions, and PA and } \\
\text { fruit and vegetable } \\
\text { consumption self- } \\
\text { efficacy } \\
(++)\end{array}$ \\
\hline
\end{tabular}


Table 1 Overview of the publications included in the review (Continued)

\begin{tabular}{|c|c|c|c|c|c|c|c|c|}
\hline Publication & $\begin{array}{l}\text { Program } \\
\text { name }\end{array}$ & Country & $\begin{array}{l}\text { Population } \\
\text { group }\end{array}$ & Setting & $\begin{array}{l}\text { Intervention } \\
\text { duration }\end{array}$ & $\begin{array}{l}\text { Intervention } \\
\text { Approach \& } \\
\text { Theoretical } \\
\text { framework }\end{array}$ & $\begin{array}{l}\text { Post-trial } \\
\text { follow-up } \\
\text { duration }\end{array}$ & $\begin{array}{l}\text { Outcomes \& } \\
\text { Effectiveness score }\end{array}$ \\
\hline $\begin{array}{l}\text { Weber et al., } \\
2017 \text { [49] }\end{array}$ & $\begin{array}{l}\text { SMS. } \\
\text { Sei schlau. } \\
\text { Mach mit. Sei } \\
\text { fit. } \\
\text { ['Be smart. } \\
\text { Join in. Be } \\
\text { fit.'] }\end{array}$ & Germany & $\begin{array}{l}\text { Four 3rd and } \\
4 \text { th grade } \\
\text { classes ( } 70 \\
\text { children) as } \\
\text { intervention } \\
\& \\
6 \text { classes } \\
\text { (125 } \\
\text { children) as } \\
\text { control } \\
\text { group } \\
\text { Mean age: } \\
9 \text { years }\end{array}$ & $\begin{array}{l}\text { migration } \\
\text { background }\end{array}$ & $\begin{array}{l}1 \text { school } \\
\text { year }\end{array}$ & $\begin{array}{l}2 \text { additional } \\
\text { exercise lessons } \\
\text { weekly ("Fitness für } \\
\text { Kids") and } 10 \\
\text { nutrition lessons } \\
\text { per school year. } \\
\text { In the trial, parental } \\
\text { involvement was } \\
\text { limited to } \\
\text { participation in } \\
\text { evening meetings } \\
\text { and accompanying } \\
\text { their children to } \\
\text { extra-curricular } \\
\text { activities. } \\
\text { Theory Framework } \\
\text { not mentioned }\end{array}$ & - & $\begin{array}{l}\text { Significant favorable } \\
\text { effect on fitness } \\
\text { and motor skill } \\
\text { driven by higher } \\
\text { improvements in } 5 \\
\text { of the } 8 \text { test items, } \\
\text { i.e., obstacle race } \\
\text { (speed), standing } \\
\text { long jump } \\
\text { (strength), sit-ups } \\
\text { (strength), stand } \\
\text { and reach (mobil- } \\
\text { ity), and } 6 \text { min run } \\
\text { (endurance), inde- } \\
\text { pendently of } \\
\text { confounders. } \\
\text { No effects on } \\
\text { dietary knowledge } \\
\text { and consumption } \\
\text { frequencies. } \\
\text { (+) }\end{array}$ \\
\hline $\begin{array}{l}\text { Xu et al., } 2015 \\
\text { [50] }^{\mathrm{a}}\end{array}$ & $\begin{array}{l}\text { CLICK-Obesity } \\
\text { Study }\end{array}$ & $\begin{array}{l}\text { Nanjing City, } \\
\text { Mainland } \\
\text { China }\end{array}$ & $\begin{array}{l}8 \text { urban } \\
\text { primary } \\
\text { schools } \\
\text { grade } 4 \\
1125 \\
\text { students } \\
\text { Mean age: } \\
10 \text { years }\end{array}$ & & $\begin{array}{l}1 \text { school } \\
\text { year }\end{array}$ & $\begin{array}{l}\text { Classroom } \\
\text { curriculum, } \\
\text { School } \\
\text { environment } \\
\text { support, Family } \\
\text { involvement and } \\
\text { fun programs/ } \\
\text { events) together } \\
\text { with routine health } \\
\text { education } \\
\text { Theory of Triadic } \\
\text { Influence (TTI) and } \\
\text { the CDC's } \\
\text { ecological model- } \\
\text { based Coordinated } \\
\text { School Health } \\
\text { Program }\end{array}$ & & $\begin{array}{l}\text { Marginal (non- } \\
\text { significant) } \\
\text { favorable effect on } \\
\text { mean BMl value. } \\
\text { Significant } \\
\text { favorable effect on } \\
\text { likelihood to } \\
\text { decrease their BMl } \\
\text { by } 0.5 \mathrm{~kg} / \mathrm{m} 2 \text { or } \\
\text { above, increase the } \\
\text { frequency of } \\
\text { jogging/ running, } \\
\text { decrease the } \\
\text { frequency of TV/ } \\
\text { computer use and } \\
\text { of red meat } \\
\text { consumption, } \\
\text { change } \\
\text { commuting mode } \\
\text { to/from school } \\
\text { from sedentary to } \\
\text { active mode, and } \\
\text { be aware of the } \\
\text { harm of selected } \\
\text { obesity risk factors. } \\
\text { (++) }\end{array}$ \\
\hline
\end{tabular}

${ }^{a}$ The studies were included at the 2nd literature search (February 2015-January 2019), after the Feel4Diabetes intervention development Abbreviations: PE physical education; PA physical activity; EBRBs energy-balance related behaviors; CDC Centers for Disease Control; SES socioeconomic status; SDS standard deviation scores; $P E$ physical education

fruits, the availability of only healthy options in the school cafeteria and the removal of vending machines seem to be efficacious strategies. The formation of a wellness council (by school staff) and a written wellness policy were reported as some more drivers of the intervention's effectiveness.

The involvement of the family was a prerequisite for the inclusion of studies in the current review. Parents were approached via several methods, i.e. family "homework" assignments, educational/ informative material (newsletters) including healthy recipes and school meetings/ events. In all cases the aim was to encourage changes at the home environment as well in order to further promote the targeted behaviors (e.g. availability of fruit and vegetables) and ensure the continuity of the intervention after school hours.

Several other strategies were also implemented in the childhood obesity prevention interventions that were found to be effective. More specifically, physical activity promotion during recess and the development of an 
active playground as well as non-competitive, enjoyable activities, promoting whole class participation during physical education (PE) classes and the provision of additional hours to physical activity were shown to be effective strategies. Motivational incentives (e.g. stickers, t-shirts) as well as social marketing techniques targeting children, e.g. slogans, characters, raffle tickets have also been shown to contribute to effectiveness of the interventions. The collaboration with community stakeholders, local community and school policy and media in every aspect of a health promotion project targeting physical activity, healthier diet or both has been also highlighted as an important contributor to the implementation fidelity, and thus effectiveness, of the intervention, also providing the basis for a sustainable approach.

Regarding intervention programs delivered in vulnerable groups and/ or low-SES areas, effective strategies focused on school policy and environmental changes, parental engagement, incentives and interactive activities such as cooking lessons and cultural dance classes, with less emphasis given on the educational part of the intervention. Furthermore, an intervention program particularly targeting Jewish population offered gender separated physical activity classes for pupils, adapting the intervention to the needs of the specific population group thus increasing adherence and effectiveness of the intervention.

\section{Theoretical frameworks}

Various behavioral theories, models and frameworks have been utilized for the development of the studied school-based interventions. More specifically: the Social Cognitive Theory, the Theory of Planned Behavior, the Theory of Reasoned Action, the Ecological model, the Social-Ecological model, the Coordinated School Health Program model developed by the Centers for Disease Control and Prevention (CDC), Social Marketing, Intervention Mapping, the Precede-Proceed Model, as well as the Research, Vision, Action and Change methodology or a combination of the above were used as the theoretical basis for interventions' development. On the other hand, about half of the projects (11 out of 27) did not mention whether their intervention was based on a theoretical model. Based on the current review, the most effective theories used in the development of health promotion programs in the school setting are the Social Cognitive Theory and the CDC's ecological model-based Coordinated School Health Program which is commonly used in the US.

\section{Discussion}

The current review aimed to gather and evaluate available school-based, family-involved obesity prevention interventions targeting dietary, physical activity and sedentary behaviors among primary schoolchildren and their families. Moreover, it aimed to identify the most effective strategies in improving those EBRBs and tackling obesity in primary school-aged children with special emphasis given on low-SES and vulnerable groups.

Previous reviews on the same field were used as a basis for the current review and the inclusion criteria applied $[18,22,24]$. In order to avoid repetition of already proven to be effective strategies and in order to extract new meaningful results from the existing literature, the current review goes one step further in the identification of effective strategies by incorporating those already known to be effective as inclusion criteria. From the total number of studies selected 27 intervention studies (33 publications) fulfilled the inclusion criteria and were included in the current analysis.

As previously described, primary schools seem to be the ideal setting for childhood obesity prevention interventions since it offers many opportunities for physical activity promotion and nutrition education and reduction of sedentary behavior through practice, policy, and a supportive environment [18]. This approach requires the active involvement and participation of teachers. Since schools mainly focus on academic achievement, many teachers find it difficult to include extracurricular modules into the already tight schedule. Furthermore, not all teachers are willing to act as champions for a health promotion intervention, especially if their own lifestyle habits do not follow/ agree with the healthy lifestyle messages delivered via the intervention [25]. Strong engagement by the school headmaster/ leadership is crucial in order to achieve engagement of families and teachers [26] and ensure the delivery of the intervention with the highest possible fidelity and thus, effectiveness as well as teachers' behavior as role-models.

Parental influence on children's nutrition and physical activity habits is a well-known determinant of childhood obesity [27]. Based on research indicating that the involvement of parents in addressing nutrition, physical activity and sedentary behavior in children is essential, the current review only included interventions that involved parents [18, 22]. Several effective strategies such as including parents via family "homework" assignments, educational/ informative material (newsletters) and school meetings/ events have been used to involve parents in the intervention. Meetings with parents at school seemed to lead to increased effectiveness in more cases in comparison to written material, i.e. newsletters.

Given that vulnerable groups and/ or people living in low-SES areas are more likely to be overweight or obese, have poorer health outcomes, and tend to benefit less from interventions in comparison with their counterparts from more affluent backgrounds, both developed 
and developing countries should also target low-income, high-risk groups [28]. Tailoring interventions to fit the needs of a specific population group, as done in the study by Benjamins et al. where gender separated physical activity classes were offered for Jewish pupils, has been shown to be imperative for the intervention's acceptability and effectiveness [28]. A thorough situational analysis and formative research should be included in the time planning for the development of interventions, especially when targeting schools in low-SES areas and vulnerable groups [28]. The results of the current review are in agreement with previous work highlighting that intervention programs delivered in vulnerable groups and/ or low-SES areas should focus on school policy and environmental changes and interactive activities with less emphasis on the educational part of the intervention [28,29].

Using a solid theoretical framework as a basis in an intervention's design is vital to its chances of success, as evidence suggests that such interventions are more effective compared to those that are not based on a theory [30]. Various theoretical frameworks have been utilized by the included interventions, while almost half of the projects did not mention the use of a theoretical model, a fact that might have limited their effectiveness. CDC's ecological model-based Coordinated School Health Program which is commonly used in the US was one of the two more popular theories used in the development of health promotion programs in the current review. Its significant effect might derive from the fact that among several targets and in contrast to most frameworks it also aims at improving employee wellness. This characteristic might have made a difference for its popularity and effectiveness. One the other hand, the Social Cognitive Theory is a commonly used basis for interventions design. In the current review, even though it was popular in the current review, its effectiveness varied. Last but not least, integrating policies and/ or environmental changes, such as increased availability and accessibility of healthy choices and removal of unhealthy choices but also the collaboration with community stakeholders, have been shown to be a high impact intervention strategies [31,32], especially when targeting lower SES groups.

Several outcomes have been considered to assess the interventions' effectiveness in various studies. Both behaviors and clinical outcomes where considered in the included studies, however the interventions did not always succeed in having an effect on clinical outcomes. The most commonly used and assessed behavioral outcome was the consumption of fruits and vegetables and many interventions succeeded in increasing their consumption followed by physical activity, which proved to be more difficult for the interventions to significantly improve. On the other hand, BMI was the most commonly used clinical outcome assessed. Several interventions succeeded in improving BMI, while some that did not showed an effect on other important adiposity measures, i.e. waist circumference of skinfold thickness. Still, having a significant effect on clinical outcomes is not always feasible even in well-designed interventions, especially in this age group.

\section{Strengths and limitations}

The results of the current work should be interpreted in consideration of the study's strengths and limitations. In the current review studies in languages other than English were omitted, which could limit the generalization of the results in other countries, especially low-to-middle income countries (LMICs). Studies without published outcomes were excluded as it was not possible to evaluate their efficacy. Although the benefits of single health behavior change interventions vs. multiple health behavior change interventions remain unclear [33], the present review only included interventions targeting dietary, physical activity and sedentary behaviors simultaneously. On the other hand, the current review goes beyond the existing literature by applying specific inclusion criteria that have been linked to increased effectiveness and thus, focusing on the most effective programs to extract the best intervention strategies, contributing to the updating of the literature.

\section{Conclusions}

School-based interventions are vital in the prevention of the globally rising childhood obesity. Many interventions have shown promising results, which were supported by a number of effective and high-impact strategies. Multiple strategies are used in effective interventions, highlighting the fact that a one-size-fits-all approach is not applicable in childhood obesity prevention intervention programs development and that many different strategies can be effective. However, future school-based obesity prevention interventions should build on already successful intervention strategies while also addressing and integrating culture specific strategies. Including long-term follow-up measurements to assess the efficacy of school-based interventions will facilitate the identification of the most effective strategies in the long-term.

Further studies are needed to elucidate the effectiveness of specific strategies aiming at long duration interventions. Both the development and the implementation methodology of the research, as well as the barriers, challenges and possible facilitators should be encouraged to be thoroughly recorded and published in order to inform the scientific community on the feasibility and sustainability of implementing interventions in real life situations.

\section{Recommendations for future school-based obesity prevention interventions}

Apart from the already mentioned recommendations that have been used as inclusion criteria in the present 
review, several strategies have been proven efficacious, i.e.:

- The use of a suitable theoretical framework should be considered in the intervention design

- Interventions should be adapted to the needs of the targeted population group(s)

- Collaboration with community stakeholders, local policy and media should be targeted in order to increase intervention's acceptability and sustainability

- School teachers should act as role-models and get actively involved in all targeted behaviors

- The teaching staff and school personnel should promote the availability and accessibility of healthy snacks and water and monitor the provision only of healthy food options by the school cafeteria/canteen and attend to the removal of unhealthy choices and vending machines, if any

- Schools should be encouraged to form a wellness council (school staff and/or parents' association) and write a wellness policy

- Families should be targeted via educational/ informative material (newsletters), family "homework" assignments or school meetings/ events for the children and their families in order to change the home environment to promote the targeted behaviors (e.g. availability of fruit and vegetable, as well as other healthy food items/snacks)

- School yards should be accessible after school hours and physical activity during recess should be promoted

- PE-instructor/school teachers should develop and promote non-competitive, enjoyable activities, promoting whole class participation

- Social marketing techniques, interactive activities and motivational incentives can be utilized for children.

- In low-SES areas or in migrant population groups, interventions should focus on school policy and environmental changes, parental engagement and interactive team activities, rather than the educational part of the intervention.

\section{Supplementary information}

Supplementary information accompanies this paper at https://doi.org/10. 1186/s12902-020-0526-5.

Additional file 1: Table 1 PICO keywords.

\section{Abbreviations}

BMI: Body mass index; CDC: Centers for Disease Control and Prevention; EBRBs: Energy balance related behaviors; HICs: High Income Countries; LMICs: Low-to-Middle Income Countries; SES: Socio-economic status; PE: Physical education

\section{Acknowledgements}

The authors would like to thank the members of the Feel4Diabetes-study group: Coordinator: Yannis Manios Steering Committee: Yannis Manios, Greet Cardon, Jaana Lindström, Peter Schwarz, Konstantinos Makrilakis, Lieven Annemans, Ignacio Garamendi Harokopio University (Greece) Yannis Manios, Kalliopi Karatzi, Odysseas Androutsos, George Moschonis, Spyridon Kanellakis, Christina Mavrogianni, Konstantina Tsoutsoulopoulou, Christina Katsarou, Eva Karaglani, Irini Qira, Efstathios Skoufas, Konstantina Maragkopoulou, Antigone Tsiafitsa, Irini Sotiropoulou, Michalis Tsolakos, Effie Argyri, Mary Nikolaou, Eleni-Anna Vampouli, Christina Filippou, Kyriaki Apergi, Amalia Filippou, Gatsiou Katerina, Efstratios Dimitriadis National Institute for Health and Welfare (Finland) Jaana Lindström, Tiina Laatikainen, Katja Wikström, Petteri Hovi, Jemina Kivelä, Päivi Valve, Esko Levälahti, Eeva Virtanen Ghent University (Belgium) Department of Movement and Sports Sciences: Greet Cardon, Vicky Van Stappen, Nele Huys Department of Public Health: Lieven Annemans, Ruben Willems Department of Endocrinology and Metabolic Diseases: Samyah Shadid Technische Universität Dresden (Germany) Peter Schwarz, Patrick Timpel University of Athens (Greece) Konstantinos Makrilakis, Stavros Liatis, George Dafoulas, Christina-Paulina Lambrinou, Angeliki Giannopoulou International Diabetes Federation European Region (Belgium) Lala Rabemananjara, Maria Stella de Sabata, Winne Ko, Ignacio Garamendi Universidad De Zaragoza (Spain) Luis Moreno, Fernando Civeira, Gloria Bueno, Pilar De Miguel-Etayo, Esther Ma Gonzalez-Gil, María L. Miguel-Berges, Natalia Giménez-Legarre; Paloma Flores-Barrantes, Aleli M. Ayala-Marín, Miguel SeralCortés, Lucia Baila-Rueda, Ana Cenarro, Estíbaliz Jarauta, Rocío Mateo-Gallego Medical University of Varna (Bulgaria) Violeta lotova, Tsvetalina Tankova, Natalia Usheva, Kaloyan Tsochev, Nevena Chakarova, Sonya Galcheva, Rumyana Dimova, Yana Bocheva, Zhaneta Radkova, Vanya Marinova, Yuliya Bazdarska, Tanya Stefanova University of Debrecen (Hungary) Imre Rurik, Timea Ungvari, Zoltán Jancsó, Anna Nánási, László Kolozsvári, Csilla Semánova, Éva Bíró, Emese Antal, Sándorné Radó Extensive Life Oy (Finland) Remberto Martinez, Marcos Tong.

\section{About this supplement}

This article has been published as part of BMC Endocrine Disorders, Volume 20 Supplement 2, 2020: Designing, implementing and evaluating a community-based intervention to prevent diabetes in vulnerable families across Europe. The Feel4Diabetes-study. The full contents of the supplement are available at https://bmcendocrdisord.biomedcentral.com/articles/ supplements/volume-20-supplement-2.

\section{Authors' contributions}

$\mathrm{CPL}$ and $\mathrm{OA}$ constructed the search strategy for review and they also read and selected articles. CPL, OA, EK (HUA) and YM wrote the first draft of the manuscript. GC, NH, KW, JK, WK, EK (IDFE), KT, VI, RD, DMEP, GGEM, HT, ZJ, SL and $\mathrm{KM}$ critically revised the manuscript and read and approved the final manuscript.

\section{Funding}

The Feel4Diabetes-study has received funding from the European Union's Horizon 2020 research and innovation programme [Grant Agreement: ${ }^{\circ}$ 643708]. The content of this article reflects only the authors' views and the European Community is not liable for any use that may be made of the information contained therein.

Availability of data and materials

Not applicable.

Ethics approval and consent to participate

This study was conducted according to the guidelines laid down in the Declaration of Helsinki and all procedures involving human subjects were approved by the ethics committees in all countries (in Belgium, by the Medical Ethics Committee of the Ghent University Hospital; in Bulgaria, by the Ethics Committee of the Medical University of Varna; in Finland, by the hospital district of Southwest Finland ethical committee; in Greece, by the Bioethics Committee of Harokopio University; in Hungary, by the National Committee for Scientific Research in Medicine; in Spain, by the Clinical Research Ethics Committee).

Consent for publication

Not applicable. 


\section{Competing interests}

Two co-authors are members of the editorial board (Associate Editor - Odysseas Androutsos, Section Editor- Stavros Liatis) of this journal. The authors declare that they have no further competing interests.

\section{Author details}

'Department of Nutrition and Dietetics, School of Health Science and Education, Harokopio University, 70 El Venizelou Ave, 17671 Kallithea, Athens, Greece. ${ }^{2}$ Department of Nutrition and Dietetics, School of Physical Education, Sport Science and Dietetics, University of Thessaly, Trikala, Greece. ${ }^{3}$ Department of Movement and Sports Sciences, Faculty of Medicine and Health Sciences, Ghent University, Ghent, Belgium. ${ }^{4}$ Department of Public Health Solutions, National Institute for Health and Welfare, Helsinki, Finland. ${ }^{5}$ International Diabetes Federation European Region, Brussels, Belgium. ${ }^{6}$ Department of Pediatrics, Medical University Varna, Varna, Bulgaria. ${ }^{7}$ Department of Diabetology, Clinical Center of Endocrinology, Medical University Sofia, Sofia, Bulgaria. ${ }^{8}$ Growth, Exercise, NUtrition and Development (GENUD) Research Group. Instituto Agroalimentario de Aragón (IA2), Instituto de Investigación Sanitaria Aragón (IIS Aragón), University of Zaragoza, Zaragoza, Spain. ${ }^{9}$ Centro de Investigación Biomédica en Red de Fisiopatología de la Obesidad y Nutrición (CIBERObn), University of Zaragoza, Zaragoza, Spain. ${ }^{10}$ Institute of Nutrition and Food Technology. Department of Biochemistry and Molecular Biology II, Center of Biomedical Research, University of Granada, Granada, Spain. ${ }^{11}$ University of Debrecen, Department of Family and Occupational Medicine, Debrecen, Hungary. ${ }^{12}$ National and Kapodistrian University of Athens, Athens, Greece.

\section{Received: 14 March 2020 Accepted: 23 March 2020}

Published: 6 May 2020

\section{References}

1. Lobstein T, Baur L, Uauy R. Obesity in children and young people: a crisis in public health. Obes Rev. 2004:5:4-85.

2. Ogden $C L$, Carroll MD, Curtin LR, Lamb MM, Flegal KM. Prevalence of high body mass index in US children and adolescents, 2007-2008. Jama. 2010; 303(3):242-9.

3. Hales CM, Fryar CD, Carroll MD, Freedman DS, Ogden CL. Trends in obesity and severe obesity prevalence in us youth and adults by sex and age, 20072008 to 2015-2016. JAMA. 2018;319(16):1723-5.

4. Jackson-Leach R, Lobstein T. Estimated burden of paediatric obesity and comorbidities in Europe. Part 1. The increase in the prevalence of child obesity in Europe is itself increasing. Int J Pediatr Obes. 2006; 1(1):26-32.

5. Manios Y, Androutsos O, Katsarou C, Vampouli EA, Kulaga Z, Gurzkowska B, et al. Prevalence and sociodemographic correlates of overweight and obesity in a large Pan-European cohort of preschool children and their families: the ToyBox study. Nutrition. 2018;55-56:192-8.

6. Blundell JE, Baker JL, Boyland E, Blaak E, Charzewska J, de Henauw S, et al. Variations in the prevalence of obesity among European countries, and a consideration of possible causes. Obesity Facts. 2017;10(1):25-37.

7. Ezzati M, Lopez AD, Rodgers A, Murray CJ. Comparative quantification of health risks: global and regional burden of disease attributable to selected major risk factors: OMS; 2004.

8. Fund WCR, Research AlfC. Food, nutrition, physical activity, and the prevention of cancer: a global perspective: Amer Inst for Cancer research; 2007.

9. Organization WH. Global status report on noncommunicable diseases 2010. Geneva: World Health Organization; 2011.

10. Organization WH, Canada PHAo, Canada CPHAo. Preventing chronic diseases: a vital investment: World Health Organization; 2005.

11. Olstad DL, McCargar L. Prevention of overweight and obesity in children under the age of 6 years. Appl Physiol Nutr Metab. 2009;34(4):551-70.

12. Whitaker RC, Wright JA, Pepe MS, Seidel KD, Dietz WH. Predicting obesity in young adulthood from childhood and parental obesity. N Engl J Med. 1997; 337(13):869-73.

13. Parsons TJ, Power C, Logan S, Summerbell CD. Childhood predictors of adult obesity: a systematic review. Int J Obes Relat Metab Disord. 1999; 23(Suppl 8):S1-107.

14. Singh AS, Mulder C, Twisk JW, van Mechelen W, Chinapaw MJ. Tracking of childhood overweight into adulthood: a systematic review of the literature. Obes Rev. 2008;9(5):474-88.
15. Evensen E, Wilsgaard T, Furberg A-S, Skeie G. Tracking of overweight and obesity from early childhood to adolescence in a population-based cohort the Troms $\varnothing$ Study, Fit Futures. BMC Pediatr. 2016;16:64.

16. Pandita A, Sharma D, Pandita D, Pawar S, Tariq M, Kaul A. Childhood obesity: prevention is better than cure. Diabetes Metab Syndr Obes. 2016;9:83-9.

17. Pyle SA, Sharkey J, Yetter G, Felix E, Furlong MJ, Poston WSC. Fighting an epidemic: the role of schools in reducing childhood obesity. Psychol Sch. 2006;43(3):361-76.

18. Khambalia AZ, Dickinson S, Hardy LL, Gill T, Baur LA. A synthesis of existing systematic reviews and meta-analyses of school-based behavioural interventions for controlling and preventing obesity. Obes Rev. 2012;13(3): 214-33.

19. Manios Y, Androutsos O, Lambrinou CP, Cardon G, Lindstrom J, Annemans $L$, et al. A school- and community-based intervention to promote healthy lifestyle and prevent type 2 diabetes in vulnerable families across Europe: design and implementation of the Feel4Diabetes-study. Public Health Nutr. 2018;21(17):3281-90

20. Wang Y, Lobstein T. Worldwide trends in childhood overweight and obesity. Int J Pediatr Obes. 2006;1(1):11-25.

21. Agardh E, Allebeck P, Hallqvist J, Moradi T, Sidorchuk A. Type 2 diabetes incidence and socio-economic position: a systematic review and metaanalysis. Int J Epidemiol. 2011;40(3):804-18.

22. Sharma M. Dietary education in school-based childhood obesity prevention programs. Adv Nutr. 2011;2(2):207S-16S

23. Neil Fantom HF, William C. Prince. 2014. Available from: https://blogs. worldbank.org/opendata/lics-Imics-umics-and-hics-classifying-economiesanalytical-purposes.

24. Nixon CA, Moore HJ, Douthwaite W, Gibson EL, Vogele C, Kreichauf S, et al. Identifying effective behavioural models and behaviour change strategies underpinning preschool- and school-based obesity prevention interventions aimed at 4-6-year-olds: a systematic review. Obes Rev. 2012;13(Suppl 1):106-17.

25. Jago R, Rawlins E, Kipping RR, Wells S, Chittleborough C, Peters TJ, et al. Lessons learned from the AFLY5 RCT process evaluation: implications for the design of physical activity and nutrition interventions in schools. BMC Public Health. 2015;15:946.

26. Blaine RE, Franckle RL, Ganter C, Falbe J, Giles C, Criss S, et al. Using school staff members to implement a childhood obesity prevention intervention in lowincome school districts: the Massachusetts childhood obesity research demonstration (MA-CORD project), 2012-2014. Prev Chronic Dis. 2017;14:E03.

27. Budd GM, Hayman LL. Childhood obesity: determinants, prevention, and treatment. J Cardiovasc Nurs. 2006;21(6):437-41.

28. Coupe N, Cotterill S, Peters S. Tailoring lifestyle interventions to low socioeconomic populations: a qualitative study. BMC Public Health. 2018;18(1):967.

29. Ickes MJ, McMullen J, Haider T, Sharma M. Global school-based childhood obesity interventions: a review. Int J Environ Res Public Health. 2014;11(9): 8940-61.

30. Sharma M. International school-based interventions for preventing obesity in children. Obes Rev. 2007;8(2):155-67.

31. Lloyd J, Creanor S, Logan S, Green C, Dean SG, Hillsdon M, et al. Effectiveness of the healthy lifestyles Programme (HeLP) to prevent obesity in UK primary-school children: a cluster randomised controlled trial. Lancet Child Adolesc Health. 2018;2(1):35-45.

32. Marild $S$, Russo $P$, Veidebaum $T$, Tornaritis $M$, De Henauw $S$, De Bourdeaudhuij I, et al. Impact of a community based health-promotion programme in 2- to 9-year-old children in Europe on markers of the metabolic syndrome, the IDEFICS study. Obes Rev. 2015;16(Suppl 2):41-56.

33. James E, Freund M, Booth A, Duncan MJ, Johnson N, Short CE, et al. Comparative efficacy of simultaneous versus sequential multiple health behavior change interventions among adults: a systematic review of randomised trials. Prev Med. 2016;89:211-23.

34. Lloyd J, Creanor S, Logan S, Green C, Dean SG, Hillsdon M, et al. Effectiveness of the Healthy Lifestyles Programme (HeLP) to prevent obesity in UK primary-school children: a cluster randomised controlled trial. Lancet Child Adolesc Health. 2018;2(1):35-45.

35. Manios Y, Moschandreas J, Hatzis C, Kafatos A. Health and nutrition education in primary schools of Crete: changes in chronic disease risk factors following a 6-year intervention programme. Br J Nutr. 2002;88(3):31524.

36. Manios Y, Kafatos I, Kafatos A. Ten-year follow-up of the Cretan Health and Nutrition Education Program on children's physical activity levels. Prev Med. 2006;43(6):442-6. 
37. Manios $Y$, Kafatos A. Health and nutrition education in primary schools in Crete: 10 years follow-up of serum lipids, physical activity and macronutrient intake. Br J Nutr. 2006;95(3):568-75.

38. Marild $S$, Russo $P$, Veidebaum $T$, Tornaritis $M$, De Henauw $S$, De Bourdeaudhuij I, et al. Impact of a community based health-promotion programme in 2- to 9-year-old children in Europe on markers of the metabolic syndrome, the IDEFICS study. Obes Rev. 2015;16(Suppl 2):41-56.

39. Mihrshahi S, Vaughan L, Fa'avale N, De Silva Weliange S, Manu-Sione I, Schubert L. Evaluation of the Good Start Program: a healthy eating and physical activity intervention for Maori and Pacific Islander children living in Queensland, Australia. BMC Publ Health. 2017;17(1):77.

40. Pablos A, Nebot V, Vano-Vicent V, Ceca D, Elvira L. Effectiveness of a schoolbased program focusing on diet and health habits taught through physical exercise. Appl Physiol Nutr Metab. 2018;43(4):331-7.

41. Plachta-Danielzik S, Pust S, Asbeck I, Czerwinski-Mast M, Langnase K, Fischer C, et al. Four-year follow-up of school-based intervention on overweight children: the KOPS study. Obesity (Silver Spring, Md). 2007;15(12):3159-69.

42. Plachta-Danielzik S, Landsberg B, Lange D, Seiberl J, Muller MJ. Eight-year follow-up of school-based intervention on childhood overweight--the Kiel Obesity Prevention Study. Obes Facts. 2011;4(1):35-43.

43. Rush E, McLennan S, Obolonkin V, Vandal AC, Hamlin M, Simmons D, et al. Project Energize: whole-region primary school nutrition and physical activity programme; evaluation of body size and fitness 5 years after the randomised controlled trial. Br J Nutr. 2014;111(2):363-71.

44. Sacchetti R, Dallolio L, Musti MA, Guberti E, Garulli A, Beltrami P, et al. Effects of a school based intervention to promote healthy habits in children 8-11 years old, living in the lowland area of Bologna Local Health Unit. Ann Ig. 2015;27(2):432-46.

45. Sahota P, Rudolf MC, Dixey R, Hill AJ, Barth JH, Cade J. Randomised controlled trial of primary school based intervention to reduce risk factors for obesity. BMJ (Clinical research ed). 2001;323(7320):1029-32.

46. Shofan Y, Kedar O, Branski D, Berry E, Wilschanski M. A school-based program of physical activity may prevent obesity. Eur J Clin Nutr. 2011;65(6): 768-70.

47. Spiegel SA, Foulk D. Reducing overweight through a multidisciplinary school-based intervention. Obesity (Silver Spring, Md). 2006;14(1):88-96.

48. Springer AE, Kelder SH, Ranjit N, Hochberg-Garrett H, Crow S, Delk J. Promoting physical activity and fruit and vegetable consumption through a community-school partnership: the effects of Marathon Kids(R) on lowincome elementary school children in Texas. J Phys Act Health. 2012;9(5): 739-53.

49. Weber KS, Sporkel O, Mertens M, Freese A, Strassburger K, Kemper B, et al. Positive Effects of Promoting Physical Activity and Balanced Diets in a Primary School Setting with a High Proportion of Migrant School Children. Exp Clin Endocrinol Diabetes. 2017;125(8):554-62.

50. Xu F, Ware RS, Leslie E, Tse LA, Wang Z, Li J, et al. Effectiveness of a Randomized Controlled Lifestyle Intervention to Prevent Obesity among Chinese Primary School Students: CLICK-Obesity Study. PloS One. 2015; 10(10):e0141421.

\section{Publisher's Note}

Springer Nature remains neutral with regard to jurisdictional claims in published maps and institutional affiliations.

Ready to submit your research? Choose BMC and benefit from:

- fast, convenient online submission

- thorough peer review by experienced researchers in your field

- rapid publication on acceptance

- support for research data, including large and complex data types

- gold Open Access which fosters wider collaboration and increased citations

- maximum visibility for your research: over $100 \mathrm{M}$ website views per year

At $\mathrm{BMC}$, research is always in progress.

Learn more biomedcentral.com/submissions 